\title{
Computational Fluid Dynamics Validation and Post-test Analysis of Supersonic Retropropulsion in the Ames $9 \times 7$ Unitary Tunnel
}

\author{
Kerry A. Zarchi (Trumble) ${ }^{*}$ \\ NASA Ames Research Center, Moffett Field, California, 94035 \\ Daniel G. Schauerhamer ${ }^{\dagger}$ \\ Jacobs Technology, NASA Johnson Space Center, Houston, Texas, 77058 \\ William Kleb ${ }^{\ddagger}$, Jan-Renee Carlson ${ }^{\S}$, and Karl Edquist ${ }^{* *}$ \\ NASA Langley Research Center, Hampton, Virginia, 23681
}

\begin{abstract}
The present paper describes an evaluation of three Navier-Stokes computational fluid dynamics codes simulating supersonic retropropulsion flow fields from a Ames 9'×7' Unitary Tunnel entry. Three codes-DPLR, FUN3D, and OVERFLOW - have been exercised (using lessons learned from a previous round of simulations) for both single- and multi-nozzle configurations for two Mach numbers and three thrust coefficients, all at zero degrees angle of attack. The focus of the present work is on high thrust coefficients and low supersonic Mach numbers, which were not considered by a previous supersonic retropropulsion wind tunnel test performed in the Langley Unitary Plan Wind Tunnel. Surface pressure measurements and shadowgraphs have been used to evaluate the flow prediction tools. All codes predict pressure measurements to within $\pm \mathbf{1 . 8 \%}$ for the smooth (nonozzle) configuration. The effect of the sting mount was also simulated to access its influence on predicted flow fields. The single- and three-nozzle configurations show periodic oscillation in triple point regions but little variation in the bow shock and plume termination shock features. The three-nozzle configuration becomes steadier with increasing thrust coefficient while the four-nozzle configurations become more chaotic. The predictions of unsteadiness and periodicity, however, do not seem to effect the predictions of time-averaged surface pressure coefficients.
\end{abstract}

\footnotetext{
* Research Scientist, Aerothermodynamics Branch, MS 230-2, Kerry.Zarchi@nasa.gov, AIAA senior member.

${ }^{\dagger}$ Aerospace Engineer, Applied Aeroscience and CFD Branch, MS EG-3, Daniel.G.Schauerhamer@nasa.gov, AIAA member.

*Aerospace Engineer, Aerothermodynamics Branch, MS 408A, Bil.Kleb@nasa.gov, AIAA lifetime member.

$\S$ Aerospace Engineer, Computational Aerosciences Branch, MS 128, Jan-Renee.Carlson@nasa.gov, AIAA senior member.

*** Aerospace Engineer, Atmospheric Flight and Entry Systems Branch, MS 489, Karl.T.Edquist@,nasa.gov, AIAA senior member.
} 


\section{Nomenclature}

\begin{tabular}{|c|c|c|c|}
\hline$M$ & $=$ Mach number & $\alpha$ & $=$ angle of attack, deg \\
\hline Re & $=$ unit Reynolds number, $\mathrm{m}^{-1}$ & $\beta$ & $=$ angle of yaw, deg \\
\hline$C_{p}$ & $=$ pressure coefficient & ( & $=$ radial angle on model, deg \\
\hline$C_{A, \text { aero }}$ & $=$ aerodynamic axial force coefficient & $\varphi$ & $=$ model roll, deg \\
\hline$C_{T}$ & $=$ thrust coefficient, $T / q_{\infty} A_{R e f}$ & $A_{\text {Ref }}$ & $=$ reference area, $0.0127 \mathrm{~m}^{2}$ \\
\hline$T$ & $=$ thrust, $\mathrm{N}$ & $\begin{array}{l}L \\
L_{r e f}\end{array}$ & $\begin{array}{l}=\text { model length, } 0.2667 \mathrm{~m} \\
=\text { reference length, } 0.127 \mathrm{~m}\end{array}$ \\
\hline \multicolumn{4}{|c|}{ Subscripts } \\
\hline$T$ & $=$ stagnation or total conditions & $R$ & $=$ model radius, $0.0635 \mathrm{~m}$ \\
\hline$\infty$ & $=$ freestream conditions & $r$ & $=$ radial coordinate, $\mathrm{m}$ \\
\hline & $=$ jet conditions & $X, Y, Z$ & $=$ model coordinate system \\
\hline
\end{tabular}

\section{Introduction}

Supersonic retropropulsion (SRP) is a potentially viable $\mathcal{N}$ alternative means for deceleration of high mass vehicles entering the Martian atmosphere. ${ }^{1,2,3,4,5,6}$ Deceleration technologies, such as parachutes, are not scalable for exploration class vehicles which are large $(>20 \mathrm{~m}$ diameter) and can potentially weigh tens of metric tons. Since ground or flight testing SRP technology at conditions relevant to Mars entry can be difficult and expensive, the advancement of this technology will depend on the ability of Computational Fluid Dynamics (CFD) to predict the flow field numerically.

Supersonic flow fields containing a mix of oncoming and counterflow streams, such as in SRP, have a complex structure involving shocks, shear layers, recirculation and stagnation regions. The concept is schematically illustrated in Figure 1 for a single nozzle configuration. The complexity of the flow with multiple physical scales (length and time) make it a challenge for flow prediction tools. The validation process chosen by the SRP team includes using multiple CFD codes to compare to historic and recent wind tunnel tests. ${ }^{7,8}$ Code-to-code and code-to-test comparisons, based on good practices in gridding, numerical method selection, and solution advancement help reduce uncertainties in the numerics, thereby allowing the focus to be on the physics of the flow fields and validity of the math models. With validation of predictive tools against ground-based tests, they can be applied more confidently to actual flight entry.

The first series of tests in the LaRC UPWT (Unitary Plan Wind Tunnel) was successful. The test series consisted of three different Mach numbers, several angles of attack and thrust coefficients for four nozzle configurations. ${ }^{10}$ The LaRC UPWT test, however, was limited in the thrust coefficient that could be reached due to size constraints of the test section. The $4{ }^{\prime} \times 4^{\prime}$ square test section experienced wall interference effects, and even tunnel blockage, for thrust coefficients greater than 3 for some

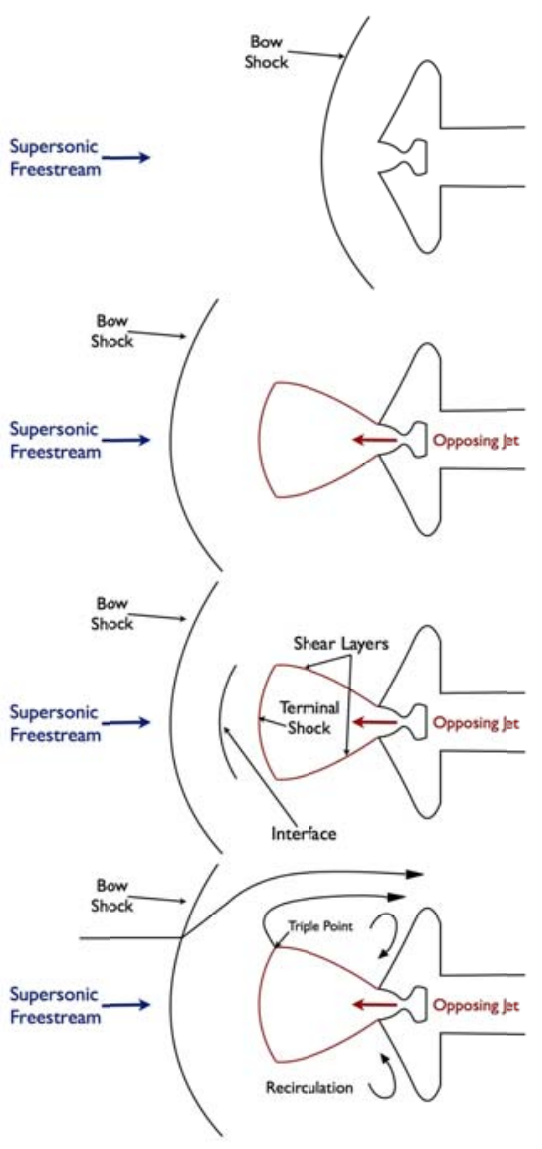

Figure 1: SRP jet plume characteristics described by Schauerhamer et $\mathbf{a l}^{8}$ for a single nozzle configuration. configurations, which was anticipated by pre-test CFD. ${ }^{11}$ The wind tunnel test in the Ames Unitary Wind Tunnel, with a $9^{\prime} \times 7^{\prime}$ test section, allowed for much higher thrust coefficients. The Ames Unitary, however, operated at lower freestream Mach numbers, 1.8 and 2.4, as 
compared to the LaRC UPWT, which was run at $M=2.4$ to 4.6. At the higher Mach number of the Ames Unitary, $M=2.4$, and multi-nozzle configurations, a maximum thrust coefficient of up to 10 was reached. Although this test does not reach flight-like thrust coefficient values, which could be on the order of $C_{T}=$ 20 , it comes closer than previously experienced. Preliminary data show that the plumes can behave much differently at these higher thrust coefficients and counter to what was observed during the LaRC UPWT test.

Understanding the aerodynamics of a vehicle experiencing SRP will contribute to the success of the mission. The objective of this paper is to apply the CFD codes at thrust coefficients greater than 3 and assess their ability in predicting surface pressure. The presence of an unsteady flow has been seen in the wind tunnel tests; and it is important to understand whether or not the ability to predict that unsteadiness will affect the ability to predict surface pressures, and hence forces and moments. Surface pressure measurements and shadowgraphs are used to evaluate the flow prediction tools. While the uncertainty quantification analysis has yet to be completed for this test, initial results are available for comparison.

\section{Computational Method}

The SRP team has applied three CFD codes to the problem: DPLR ${ }^{12}$, FUN3D ${ }^{13,14}$ and OVERFLOW. ${ }^{15}$ The codes all solve the Unsteady Reynolds-Averaged Navier-Stokes (URANS) equations, but differ in implementation, grid type, and numerical methods. Time-accurate CFD simulations have been performed due to the inherent unsteadiness of the flow fields observed in both the LaRC UPWT and Ames Unitary tests.

\section{A. DPLR}

The Data Parallel Line Relaxation (DPLR) CFD code $^{12}$ is a parallel, structured multi-block, finite volume code that solves the reacting Reynolds-Averaged Navier-Stokes equations for continuum flow, including finite rate chemistry and thermal non-equilibrium. In the present study, the thermally- and calorically-perfect URANS equations for air are solved implicitly with first-order time accuracy. The Euler fluxes are computed using modified Steger-Warming flux vector splitting ${ }^{16}$ with third-order spatial accuracy via MUSCL (Monotone Upstream-centered Schemes for Conservation Laws) extrapolation coupled with a minmod limiter. ${ }^{17}$ The viscous fluxes are computed to second-order spatial accuracy with a central difference approach. For the current analysis, the Shear-Stress Transport (SST) turbulence model was employed with a vorticity-based production term ${ }^{18}$ and no compressibility corrections. The overset gridding option available in DPLR is used extensively in the present study.

\section{B. FUN3D}

The Fully Unstructured Navier-Stokes Three-Dimensional suite of codes (FUN3D) contains a nodebased finite-volume flow solver. ${ }^{13,14}$ The FUN3D website, http://fun3d.larc.nasa.gov, contains the user manual and an extensive list of references. The present study employs Edwards' LDFSS flux function ${ }^{19}$ and a smooth Van Albada limiter ${ }^{20}$ to solve the compressible URANS equations coupled to a modified Delayed Detached Eddy Simulation model that uses the Spalart-Allmaras (SA) near-wall model. All node-based conservative variables are computed by driving a second-order accurate spatial residual to steady state with an implicit point-iterative method. An optimum second-order backward difference formula (BDF) scheme is used in conjunction with a temporal error controller that assures design order. ${ }^{21}$

\section{OVERFLOW}

OVERFLOW 2 (OVERset grid FLOW solver) is an implicit URANS flow solver that uses structured overset grids. ${ }^{15}$ For the current work, the HLLE++ numerical flux function ${ }^{22}$ with the Van Albada limiter ${ }^{20}$ was used for spatial terms, and the Symmetric Successive Over Relaxation (SSOR) algorithm with Newton subiterations for temporal terms. All viscous terms were included, and turbulence was modeled with a SSTDES hybrid model. ${ }^{23}$ The overall scheme is second-order accurate in space and time. The calculation of inviscid fluxes for both the flow solver and the turbulence model use third-order accurate MUSCL reconstruction. 


\section{Validation Wind Tunnel Test}

In Figure 2 is the 5 -inch diameter, $70^{\circ}$ sphere-cone with cylindrical aftbody wind tunnel model mounted in the $9^{\prime} \times 7^{\prime}$ test section of the NASA Ames Unitary Wind Tunnel. The same model was previously tested in the NASA Langley Unitary Plan Wind Tunnel. The nozzle configuration layout for this study is illustrated in Figure 3 for a single-, three- and four-nozzle configuration; a zero-nozzle configuration is a smooth forebody with no exposed nozzles. The model is designed with interchangeable nozzle plugs to accommodate a variety of configurations. The wind tunnel test included the four different nozzle configurations at Mach 1.8 and 2.4, at angles of attack ranging from up to $20^{\circ}$ and Reynolds number of $304,800 / \mathrm{m}$ and $457,200 / \mathrm{m}$. Thrust coefficients ranged up to 10 , with nozzle plenum pressure being the limiting factor, rather than tunnel blockage as was the case for the LaRC UPWT. Surface pressure measurements and visual information contained in shadowgraphs have been used to evaluate the flow prediction tools. The surface pressure port instrumentation layout is shown in Figure 4.

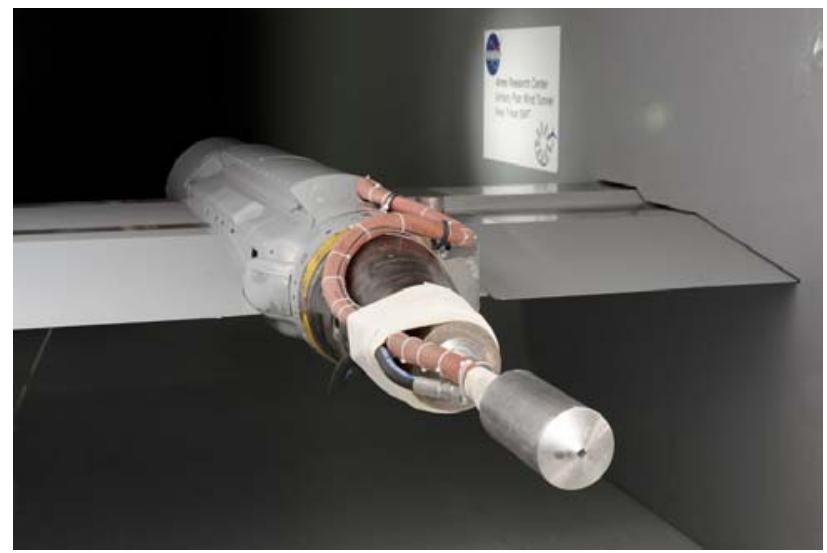

Figure 2: Mounting apparatus and 5-inch diameter wind tunnel model (single-nozzle configuration shown) in the test section of the NASA Ames 9' $\times 7^{\prime}$ Unitary Wind Tunnel. The same model was previously tested in the NASA Langley Unitary Plan Wind Tunnel.

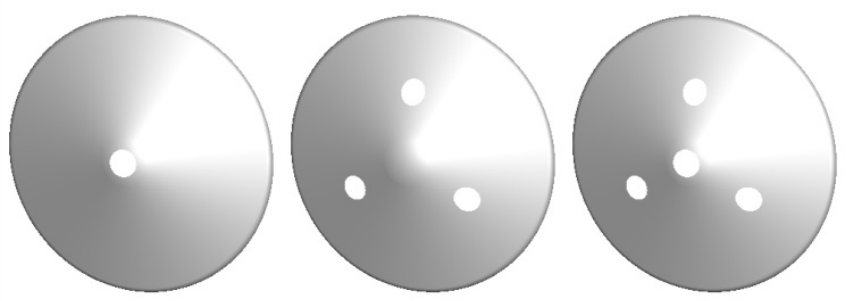

Figure 3: Nozzle configurations: single-nozzle (left), three-nozzle (center) and four-nozzle (right). 


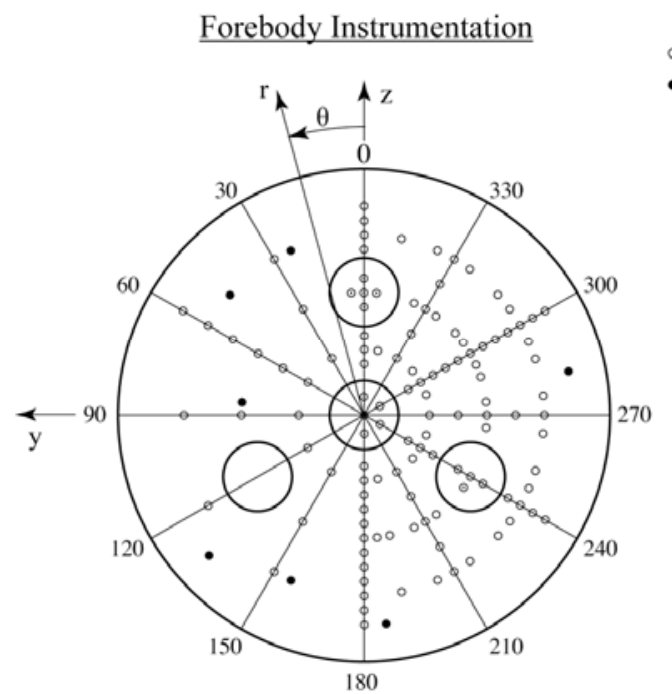

(a) Forebody of the model.
- ESP Ports Aftbody Instrumentation

- Kulites

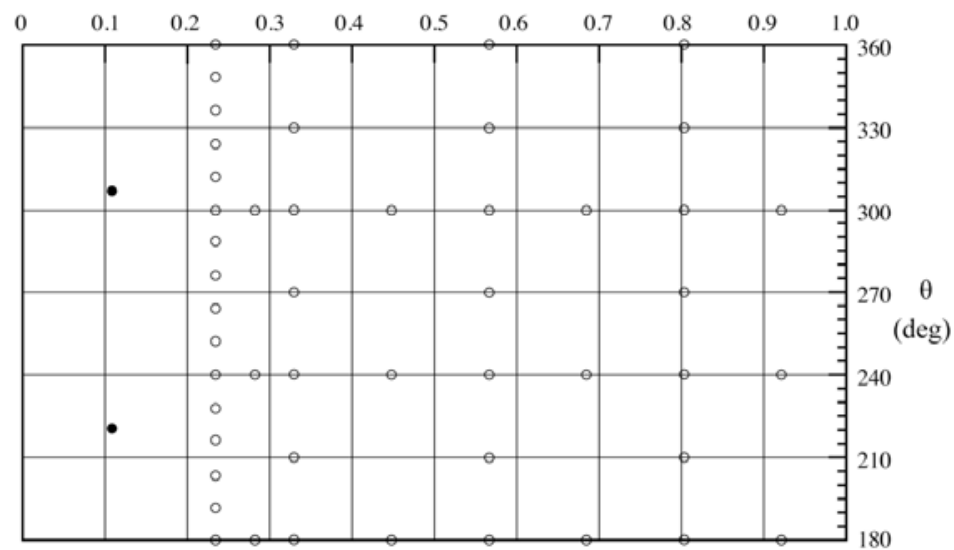

(b) Aftbody as viewed from the side of the model.

Figure 4: Pressure port (open circle) and $40 \mathrm{kHz}$ pressure transducers (filled circle) locations for the baseline configuration. Larger circles in (a) are outlines of nozzle plug locations, which can be altered to adjust for the configuration desired: $0,1,3$, or 4 nozzles.

\section{Results}

The previous work performed by the SRP CFD team was focused on establishing best practices for the codes, including grid resolution, time stepping, and turbulence modeling. ${ }^{8,9}$ This process used the LaRC UPWT test ${ }^{10}$ and the uncertainty analysis ${ }^{24}$ that came from it. The focus of the present paper, for the Ames Unitary test, is to implement these established best practices and evaluate SRP at higher thrust coefficients $\left(C_{T}>3\right)$ and lower freestream Mach numbers. The uncertainty analysis for the Ames Unitary is not yet complete. For each nozzle configuration, each freestream Mach number was evaluated at a high thrust coefficient (Table 1). All cases here are at zero angle of attack. All runs examined in this paper were tested at $R e=457,200 / \mathrm{m}$.

Table 1: Selected SRP runs from the Ames Unitary test for post-test CFD analysis.

\begin{tabular}{ccccc}
\hline \hline Run & No. of Nozzles & $\varphi(\mathrm{deg})$ & $M$ & $C_{T}$ \\
\hline 106 & 1 & 180 & 1.8 & 4 \\
116 & 1 & 180 & 2.4 & 4 \\
130 & 3 & 180 & 1.8 & 6 \\
143 & 3 & 180 & 2.4 & 8 \\
172 & 4 & 0 & 1.8 & 8 \\
179 & 4 & 0 & 2.4 & 4 \\
\hline \hline
\end{tabular}

The analysis of the Ames Unitary test began with assessing the influence of the sting on the surface pressure coefficient. Two nozzle configurations were examined to assess the presence of the sting on the CFD predictions. Next, the smooth (no-nozzle) configuration was simulated to validate the ability of CFD to predict simple flow fields within the Ames Unitary. The single-, three- and four-nozzle configurations are then examined at each freestream Mach number for high thrust coefficients.

\section{A. Sting Study}

One concern of the test was the large sting used to mount the model in the tunnel. With a subsonic pocket behind the model, it was possible that influence from the sting could travel upstream and affect pressure measurements. To quantify these possible sting effects, two run conditions, each with and without the sting, were computed using OVERFLOW to make comparisons. 
The geometry of the sting in CFD computations is not an exact representation. The geometry was developed from photographs, with some dimensions supplied by the wind tunnel staff. The sting also changed shape, in terms of both the location of the tubing as well as the shape of the casting, with every model change and roll angle increment. The present study is meant to be a first-order look into the effects of the sting, and is not an all-comprehensive quantification of the sting effects for each model configuration of the test. Figure 5 shows images of the sting and the simplified CFD model.

Two conditions, not listed in the case matrix, were simulated with and without the sting for direct comparison. The first condition was the single nozzle configuration, $C_{T}=6, M=2.4, \alpha=\beta=0^{\circ}, R e=$ $304,800 / \mathrm{m}$. The second condition was the triple nozzle configuration, $C_{T}=10, M=1.8, \alpha=\beta=0^{\circ}, R e=$ $457,200 / \mathrm{m}$. The cases were simulated with second-order time accuracy and running averages were computed. When more time steps did not change the running average of surface pressure significantly, the simulation was terminated. Perfectly steady averages were not obtained which means unsteady effects are still a contributor to differences seen between the cases.

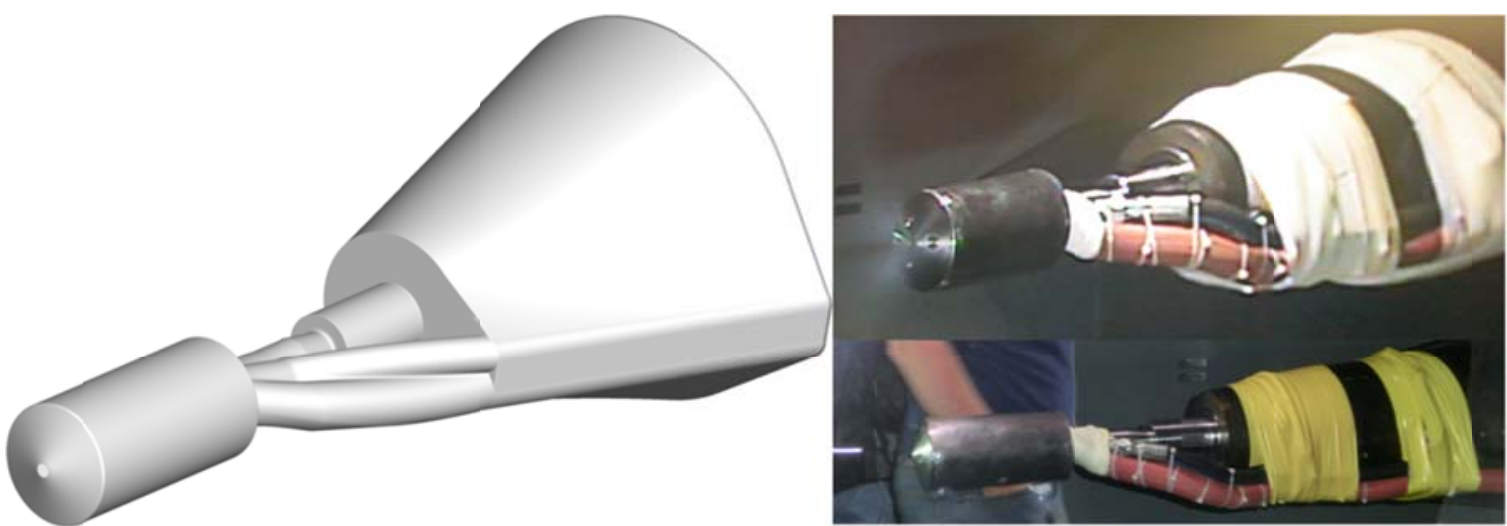

Figure 5: Sting assembly as approximated by the CFD (left) and as assembled in the Ames 9'x7' Unitary Wind Tunnel (right).

Figure 6 shows the average surface pressure of the study for the single-nozzle configuration, and Figure 7 shows the average surface pressure of the three-nozzle configuration. The images show the surface pressure coefficient averaged on the model side $(x / L)$ and forebody $(r / R)$. The two plots of averaged surface pressure show that the cases with the sting consistently predict higher pressure on the model. For both conditions, the data with the sting is closer to the test data on the model side, and for the single nozzle case, the behavior of the case with the sting closer resembles the test data. For the single nozzle case, the surface pressure on the side of the model for the sting and sting free cases meet around $x / L=0.7$, giving the impression that the sting effects do not influence upstream of that point. Overall, it can be said that sting effects are mainly confined to the aftmost pressures taps. Because of the computational expense of running these SRP cases time accurately, with turbulence modeling, and with tunnel walls, it was decided to forego the inclusion of the sting in the cases examined for this paper. It is understood that the pressure coefficient at the aft end of the model would most likely be under predicted by the CFD. 

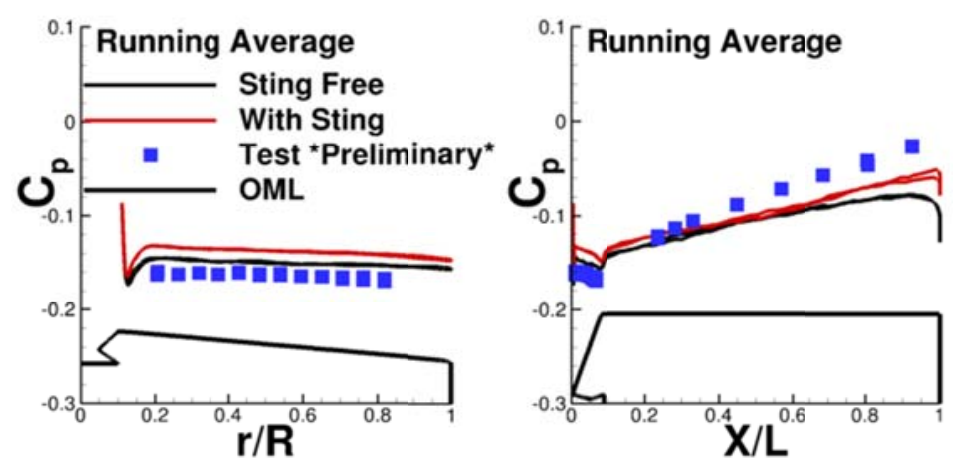

Figure 6: CFD simulation results for the single-nozzle configuration condition of the sting study. Average pressure coefficient is shown along the forebody and aftbody.
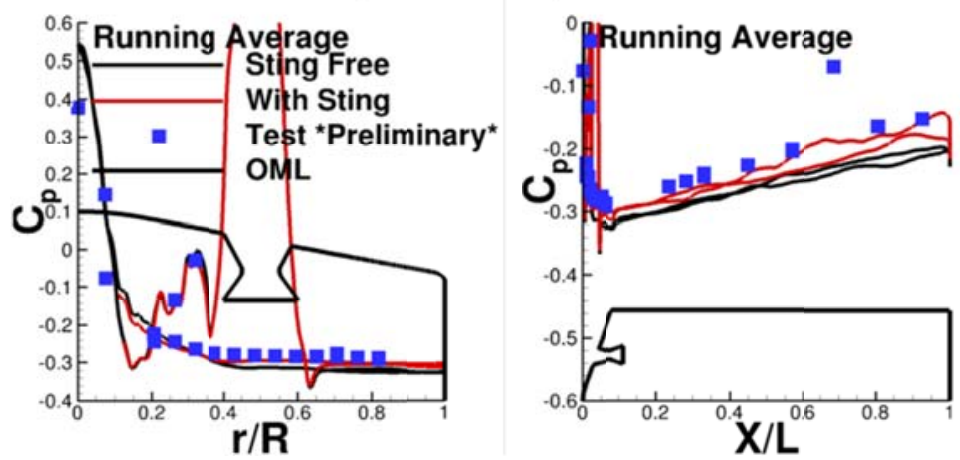

Figure 7: CFD simulation results for the three-nozzle configuration condition of the sting study. Average pressure coefficient is shown along the forebody and aftbody.

\section{B. Smooth (No-Nozzles) Configuration}

During the test a real-time assessment of the smooth (no-nozzles) configuration results, which would act as the baseline, was performed. The $M=1.8$ run had excellent agreement between the CFD codes and the experimental data (Figure 8). All sources coincided with the isentropic-compression stagnation pressure coefficient as well. The CFD from $M=2.4$ with $R e=304,800 / \mathrm{m}$ and $R e=457,200 / \mathrm{m}$ runs, however, were under predicting the experimental data by as much as $\pm 3 \%$ (Figure 9 (a) and Figure 10 (a), respectively). Run 50, which corresponds to the overlap conditions between the LaRC and Ames Unitary tests suggested an issue with the freestream Mach number reported by the Ames experiment. In the Ames UPWT experiment, the model was located further aft in the tunnel test section than is common practice, and at this location the Mach number was not well characterized. A second calibration run at the model nose location was performed, and this calibration run provided corrections to the experimental data. The corrected data brought them much closer to the CFD predicted values, within $\pm 1.8 \%$, as can been seen in Figure 9 (b) and Figure 10 (b). For more information on the corrections made to the experimental data please refer to the paper by Berry, et $\mathrm{al}^{25}$ 


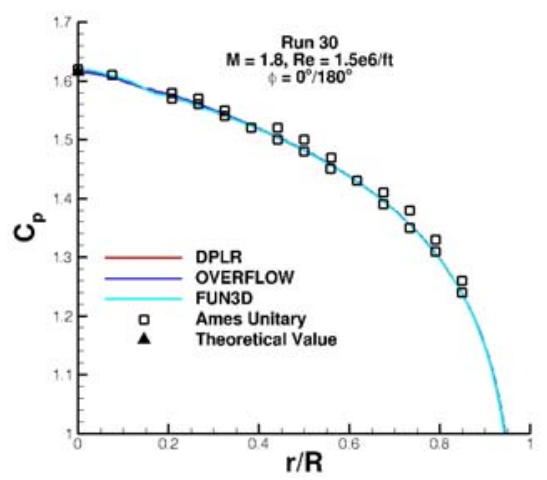

Figure 8: Pressure coefficient for the zero-nozzle configuration at Run 30.

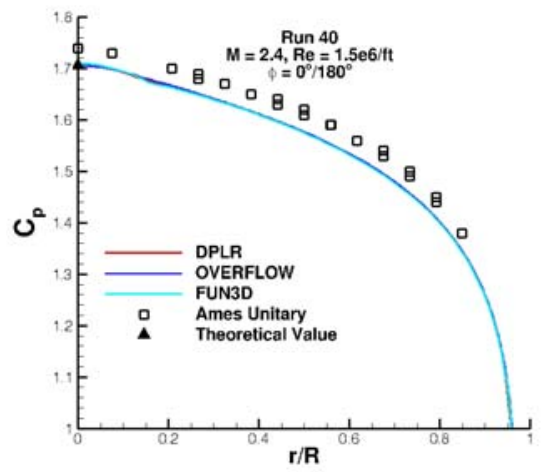

(a) Initial Wind Tunnel Data

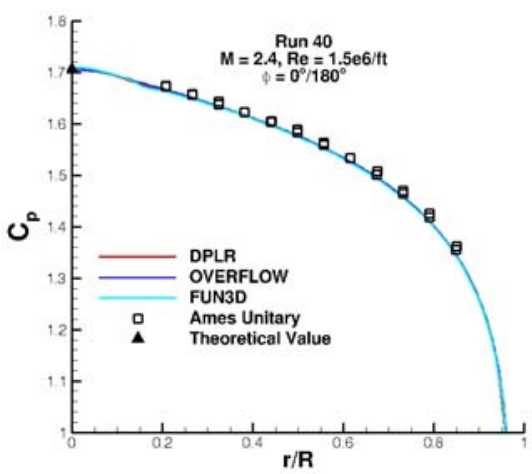

(b) Corrected Wind Tunnel Data

Figure 9: Pressure coefficient for the zero-nozzle configuration at Run 40 before (a) and after (b) corrections to the data were made.

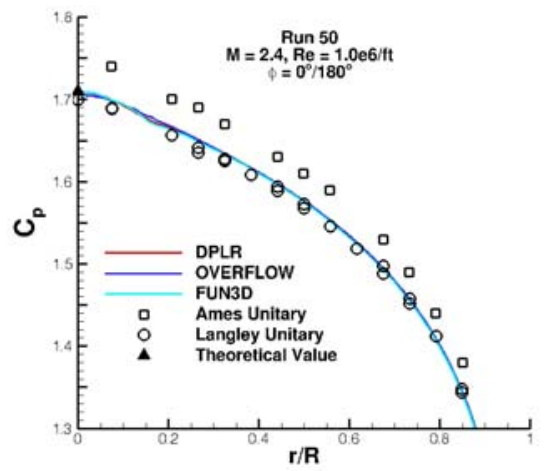

(a) Initial Wind Tunnel Data

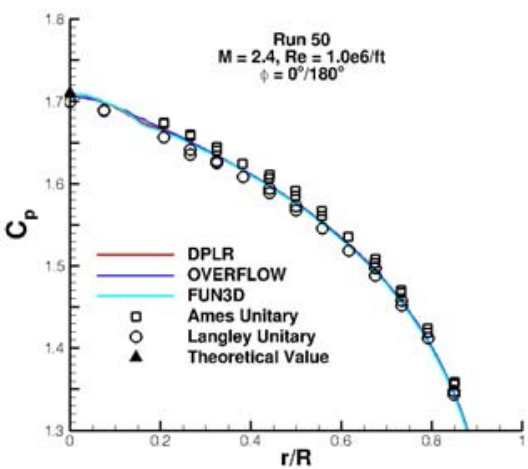

(b) Corrected Wind Tunnel Data

Figure 10: Pressure coefficient for the zero-nozzle configuration at Run 50 before (a) and after (b) corrections to the data were made. This is an overlap case with the LaRC Unitary SRP Test.

\section{Single-Nozzle Configuration}

From the LaRC UPWT tunnel test a single-nozzle configuration was used to establish the CFD code best practices. ${ }^{9}$ The case that was focused on was Run $165, M=4.6$ and $C_{T}=2$. Each code captured different variations of the unsteady oscillation of the triple point, which created pressure waves that propagated both to the model and to the bow shock. The frequencies found in the CFD codes were $2.1 \mathrm{kHz}$ for OVERFLOW and FUN3D, and $1.8 \mathrm{kHz}$ decaying toward steady for DPLR. The differences in frequencies between the codes are attributed to differences in turbulence modeling and grid refinement as shown in Ref. 9. 
For Run 106 at $M=1.8$ and $C_{T}=4$, the pressure coefficient on the forebody of the model is relatively constant, which the CFD codes capture within $\pm 5 \%$ (Figure 11 (a)). The test data lie in between the DPLR solution and the OVERFLOW and FUN3D solutions. The Run 165 from LaRC UPWT test saw positive pressure coefficient on the forebody. The negative pressure coefficient seen from the

Ames Unitary test indicates that with the higher thrust coefficient there is separation on the forebody and the plume is shielding the forebody from the freestream flow. The trend in rising pressure coefficient as $x / L$ increases across the side of the model is captured by the codes, however, at the aft end of the model the predicted pressure coefficients are lower than test data by as much as $355 \%$

(Figure 11 (b)). In

Figure 12 (a) are shadowgraphs taken at four time instances during the test are shown. These show unsteadiness in the free stagnation and triple point regions while the bow shock and plume barrel shocks remain fairly steady (features described in Figure 1). Simulated schlieren images and/or shadowgraphs extracted from the flow fields computed by the three codes are shown in

Figure 12. Instantaneous snapshots in computational time are shown to illustrate modes of unsteadiness. The lesser amount of variation of flow features within the DPLR solution is seen through the simulated shadowgraph flow field (

Figure 12 (b)), where there is little deviation between snapshots. OVERFLOW and FUN3D have a periodic oscillation at the triple point (

Figure 12 (c) and (d), respectively). The OVERFLOW and FUN3D simulated shadowgraphs show similar periodic behavior as the test. The aero component of the axial force as a function of time is shown in Figure 13. From this plot the steadier nature of the DPLR solution is reinforced, along with the periodicity of the OVERFLOW and FUN3D solutions. Dominant frequencies were found from fast Fourier transforms of the aero component of the axial force from each CFD code and are listed in Table 2, where DPLR is nearly half that of OVERFLOW and FUN3D. The differences in frequency can be attributed to DPLR modeling with a RANS turbulence model, while OVERFLOW and FUN3D are using DES turbulence models.

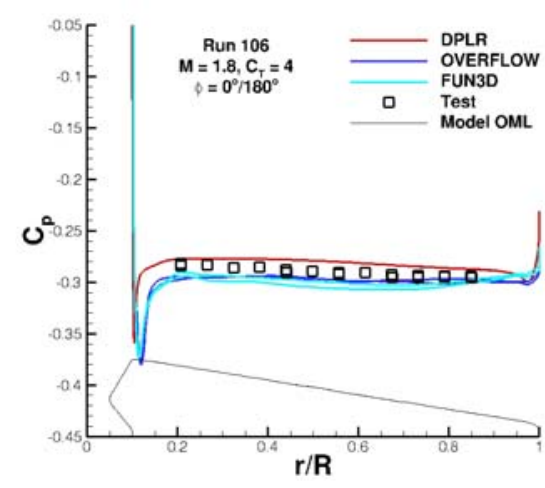

(a) Forebody

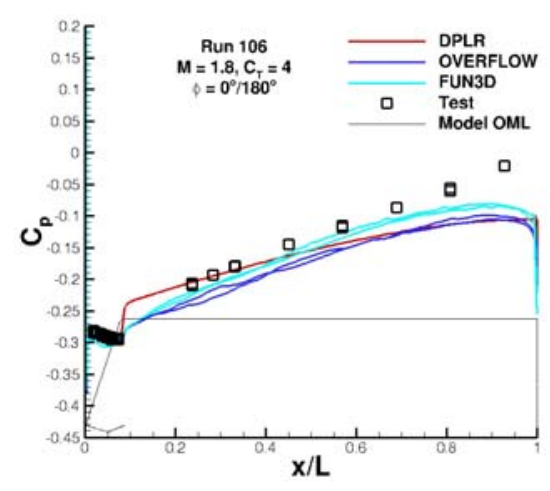

(b) Model Side

Figure 11: CFD predictions of pressure coefficients of Run 106 show excellent agreement with measurements on the forebody (a). The rise in pressure coefficient on the aft end of the model (b) is captured but not the level since CFD did not model the sting. 


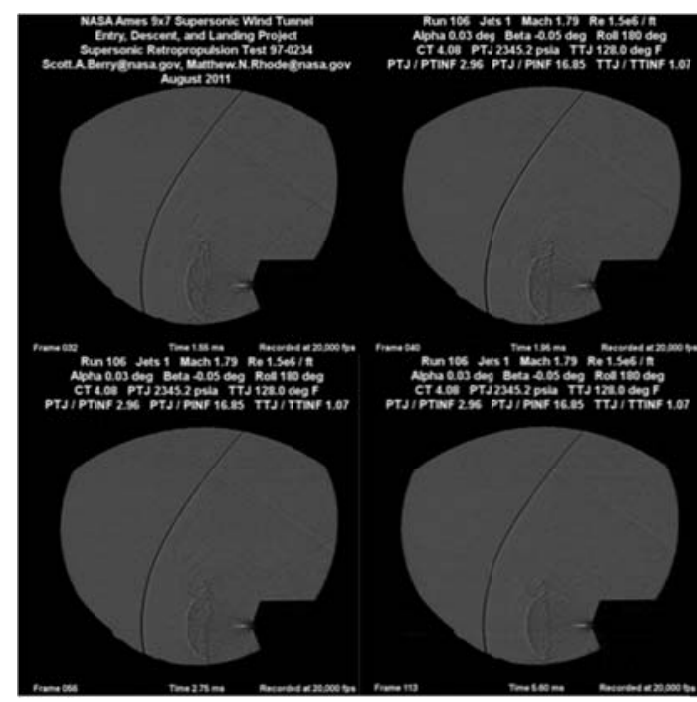

(a) Instantaneous wind tunnel shadowgraphs.
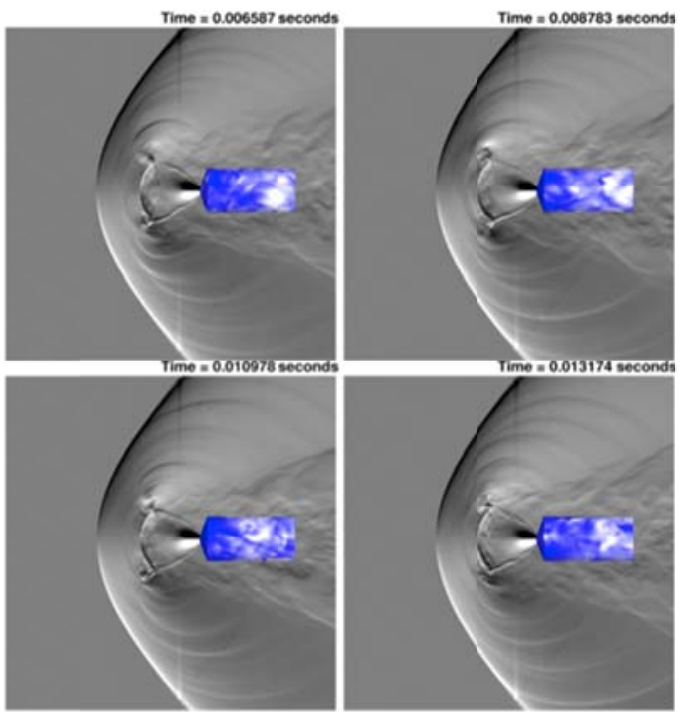

(c) Simulated schlieren from OVERFLOW flowfield solutions at four time instances.
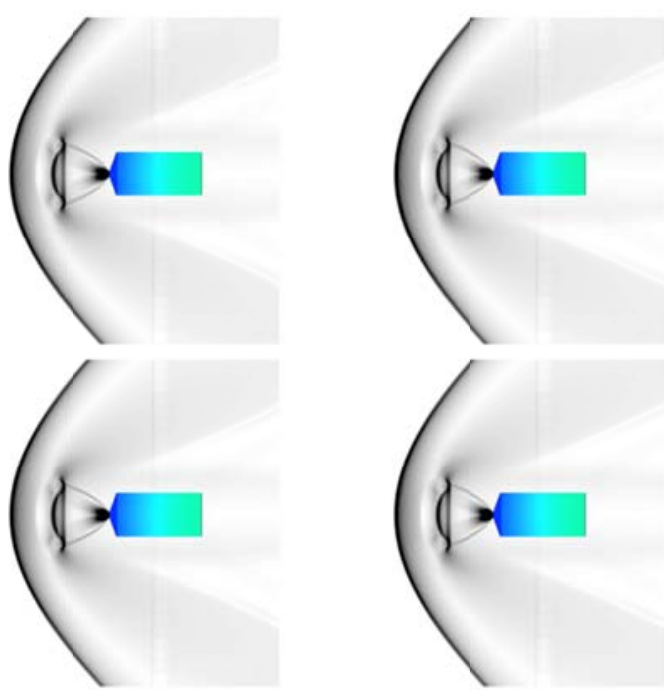

(b) Simulated shadowgraphs from DPLR flowfield solutions at four time instances.
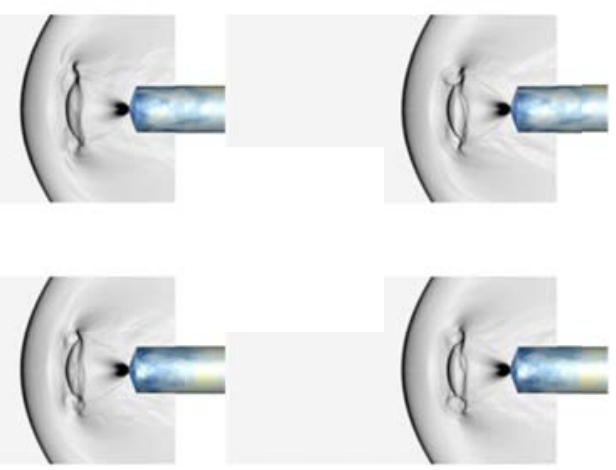

(d) Simulated shadowgraphs from FUN3D flowfield solutions at four time instances.

Figure 12: Flowfield images from wind tunnel shadowgraph and simulated shadowgraph/schlieren from CFD for Run 106. Instantaneous surface pressure coefficients are shown to qualitatively illustrate variations in time. 


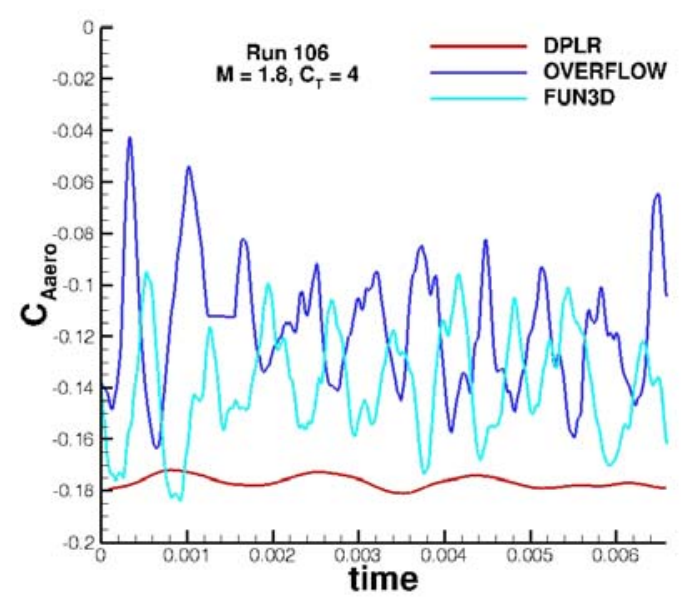

Figure 13: Aero component of transient axial force in DPLR, OVERFLOW, and FUN3D.

Table 2: Dominant frequencies in CFD codes for Run 106.

\begin{tabular}{|c|c|}
\hline Run 106 & Frequency (kHz) \\
\hline \hline DPLR & 0.60 \\
\hline OVERFLOW & 1.42 \\
\hline FUN3D & 1.38 \\
\hline
\end{tabular}

For Run 116 at $M=2.4$ and $C_{T}=4$, all codes over predict the pressure coefficient on the forebody, where the pressure coefficient remains fairly constant (Figure 14). The differences between the CFD codes ranges up to $22 \%$ and the test pressure coefficients are over predicted by as much as $24 \%$. The pressure coefficient remains negative on the forebody for this case indicating separation. Again, the trend in rising pressure coefficient as $x / L$ increases across the side of the model is captured by the codes, however, at the aft end of the model the level of pressure coefficient is under predicted by as much as $620 \%$. In

Figure 15 (a) the shadowgraphs at four time instances from the test are shown. These show a periodicity in the triple point regions while the bow shock and plume barrel shocks remain fairly steady. The steadier nature of the DPLR solution is again illustrated through the simulated shadowgraph flow field snapshots (

Figure 15 (b)) and seen in the transient axial force (Figure 16). OVERFLOW and FUN3D have a periodic oscillation at the triple point yet the bow shock and plume barrel shocks remain fairly steady (

Figure 15 (c) and (d), respectively). Simulated shadowgraphs from OVEFLOW and FUN3D solutions show similar behavior to the test indicating a good prediction of the unsteadiness. Figure 16 is the aero component of the axial force with computational time. From this plot the DPLR solution is shown to also experience a periodicity along with the FUN3D solution. The dominant frequencies were found from each CFD codes and are listed in Table 3, where DPLR is again nearly half that of OVERFLOW and FUN3D. The differences in frequency can be attributed to DPLR modeling with a RANS turbulence model, while OVERFLOW and FUN3D are modeling with DES turbulence models. The behavior between Run 106 and Run 116, where only freestream Mach number changes, is very similar. The higher Mach of Run 116 gives higher, yet still negative, pressure coefficient on the forebody and model side. This is important to understand since the controllability of a vehicle under SRP will be critical to mission success; and understanding the aerodynamics of SRP will aid in the controllability. However, the thrust has been shown to be the dominant contributor to axial force. ${ }^{8,9}$ The aero component of axial force is shown here to evaluate unsteadiness. 


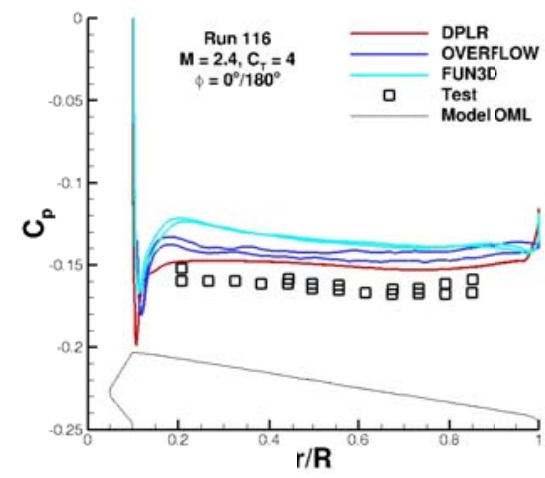

(a) Forebody

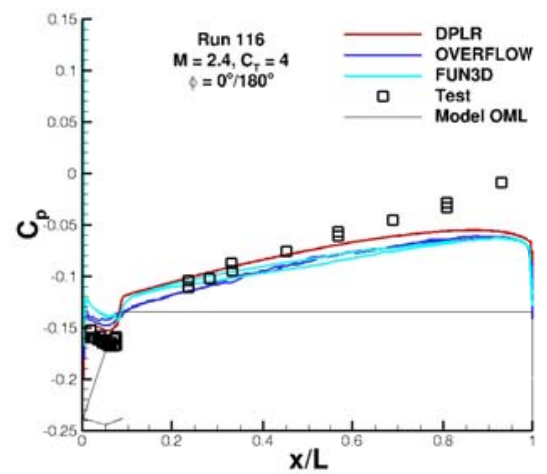

(b) Model Side

Figure 14: CFD predictions of pressure coefficients of Run 116 show excellent agreement with measurements on the forebody (a). The rise in pressure coefficient on the side (b) is captured but not the level since CFD did not model the sting.

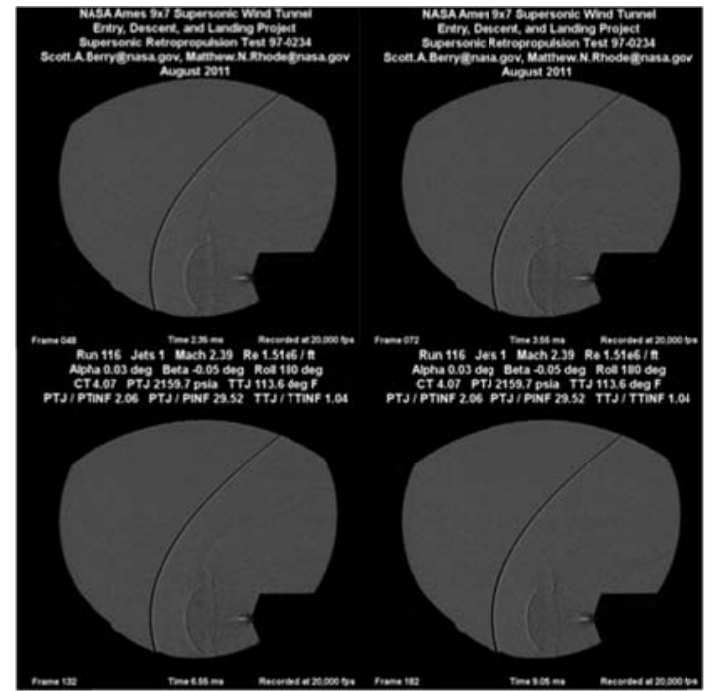

(a) Instantaneous wind tunnel shadowgraphs.

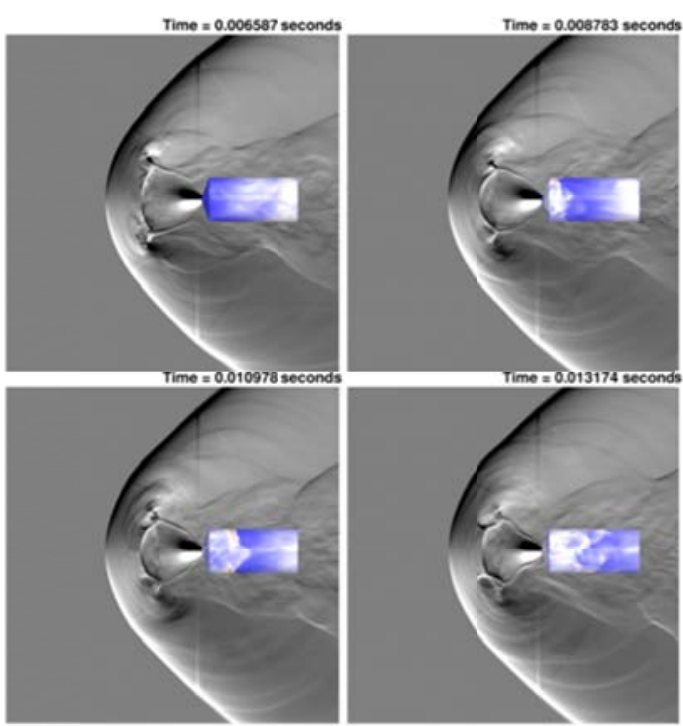

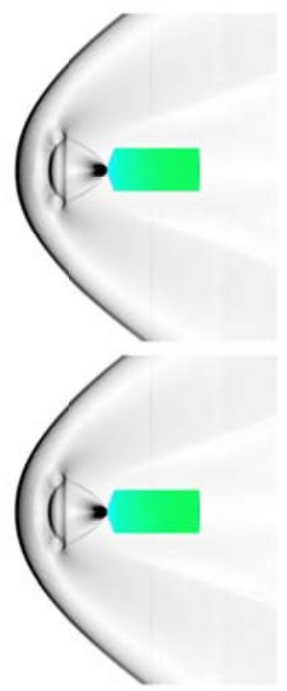

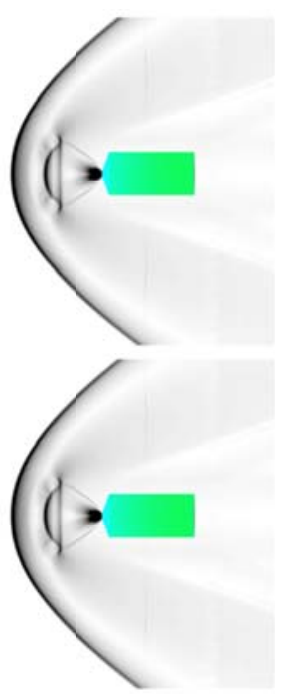

(b) Simulated shadowgraphs from DPLR flowfield solutions at four time instances.
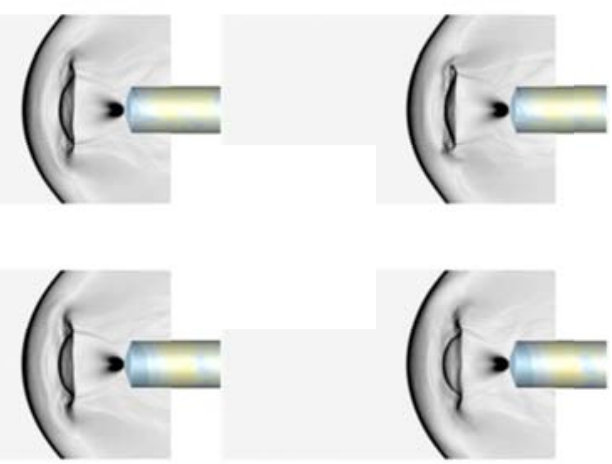
(c) Simulated schlieren from OVERFLOW

flowfield solutions at four time instances. (d) Simulated shadowgraphs from FUN3D flowfield solutions at four time instances.

Figure 15: Flowfield images from wind tunnel shadowgraph and simulated shadowgraph/schlieren from CFD for Run 116. Instantaneous surface pressure coefficients are shown to qualitatively illustrate variations in time.

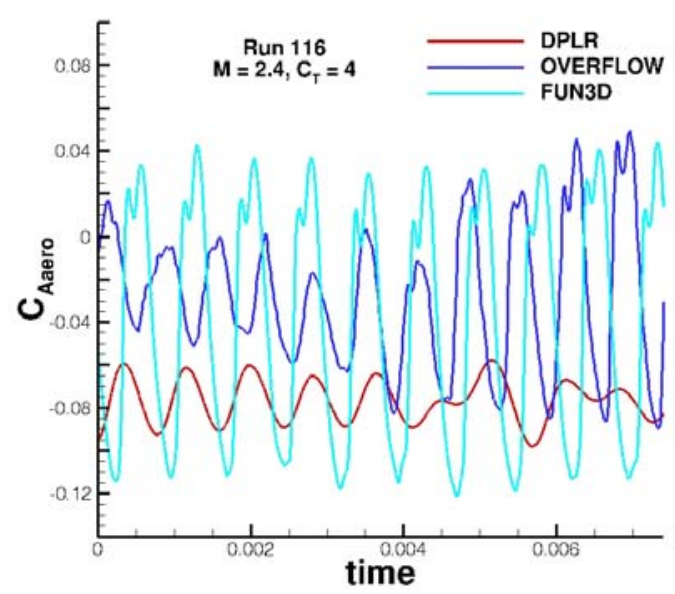

Figure 16: Aero component of transient axial force in DPLR and FUN3D.

Table 3: Dominant frequencies in CFD codes for Run 116.

\begin{tabular}{|c|c|}
\hline Run 116 & Frequency (kHz) \\
\hline \hline DPLR & 0.67 \\
\hline OVERFLOW & 1.43 \\
\hline FUN3D & 1.34 \\
\hline
\end{tabular}

\section{Three-Nozzle Configuration}

In the cases of the three-nozzle configuration, as with the single-nozzle configuration, all test data show a trend of rising pressure coefficient with increasing $x / L$ along the model sides. The CFD codes are able to capture this rising trend, but because the sting is not modeled they are under predicting the pressure coefficient at the aft end of the model. Due to the steady nature of the DPLR simulations there is very little variation in the average pressure coefficient in the radial direction, this is seen in the line plots as $\Pi=0^{\circ}$ and $\Pi=180^{\circ}$ lie nearly on top of one another. OVERFLOW and FUN3D, modeled with DES turbulence models experience higher frequencies and show variation between $\Pi$ slices.

The three-nozzle configuration cases examined using CFD for the LaRC UPWT tests were Runs 262 and $263\left(M=4.6\right.$ and $\left.C_{T}=3\right)$; the only difference in the two cases was the roll angle. The pressure coefficients seen from the LaRC UPWT test were much higher, mostly positive and near zero, than seen here in the Ames Unitary test, where they are mostly negative. The codes again have difficulty in capturing the peak pressure coefficient on the nose of the model as they did with the LaRC UPWT three-nozzle cases.

From both three-nozzle configuration cases, formation of a central feature within the region between the three plume barrel shocks is seen. This feature leads to a further thrusting out of the bow shock and steadier behavior. This observation of increased steadiness as thrust increases is counter to what was seen in the LaRC UPWT test with the three-nozzle configuration. In the earlier test, thrust coefficients were limited to below 5 due to wall interference concerns, which is approximately the point at which the flow structures tend to become steady with the Ames data. ${ }^{25}$ There was one run in the LaRC UPWT test at $M=$ 3.5, $C_{T}=6$ that was steady and further supports this claim.

In Run 130 at $M=1.8$ and $C_{T}=6$, the pressure coefficient on the forebody (Figure 17 (a)) of the model is relatively uniform on the flank with a rise at the nose, with large deviations between the codes. Differences in simulated jet expansion and jet-to-jet interactions could be the source of deviation. The CFD codes under predict the pressure coefficient on the forebody of the model by up to $52 \%$. The trend in rising 
pressure coefficient as $x / L$ increases across the side of the model is captured by the codes (Figure 17 (b)), however at the aft end of the model the level of pressure coefficient is under predicted by as much as $93 \%$. The spikes in the DPLR line plot near $x / L=0.45$ and 0.5 are an artifact of blanking from the overset regions and are not physical. In Figure 18 (a) shadowgraphs at four time instances from the test are shown. These show movement in the free stagnation and triple point regions where the bow shock and plume barrel shocks remain fairly steady. Each code shows the bow shock and plume barrel shocks remain steady with variations seen in the free stagnation and triple point regions. The steadiness of the DPLR solution is again reinforced through the simulated shadowgraphs (Figure 18 (b)). OVERFLOW (Figure 18 (c)) and FUN3D (Figure $18(\mathrm{~d})$ ) have a periodic oscillation at the triple point. The OVERFLOW and FUN3D simulated shadowgraphs show similar behavior to the test. In Figure 19 is the aero component of the axial force with computational time. From this plot the DPLR solution is shown to be completely steady and the FUN3D solution experiences some periodicity. There are many frequencies acting on this flow and it was difficult to determine the dominant frequency from the axial force for each CFD code for this case. The steady nature of the DPLR solution does not appear to adversely affect its ability to predict the average surface pressure coefficient.

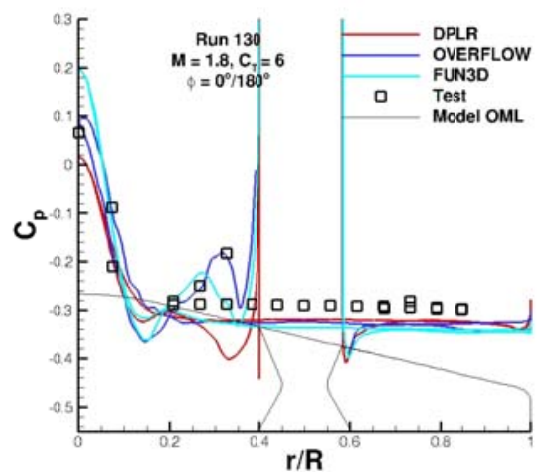

(a) Forebody

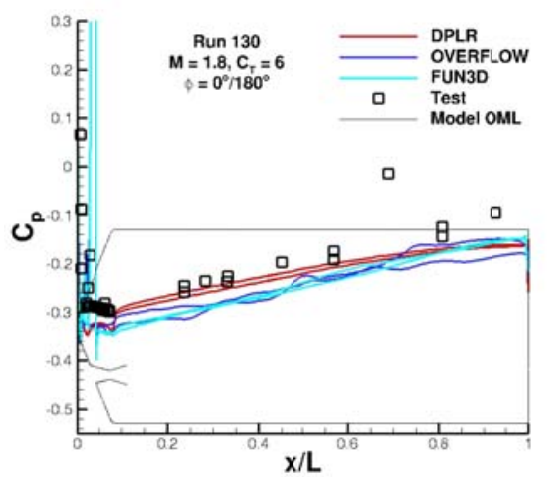

(b) Model Side

Figure 17: CFD simulations of Run 130 show excellent agreement on the forebody (a). The rise in pressure coefficient on the side (b) is captured but not the level since CFD did not model the sting.

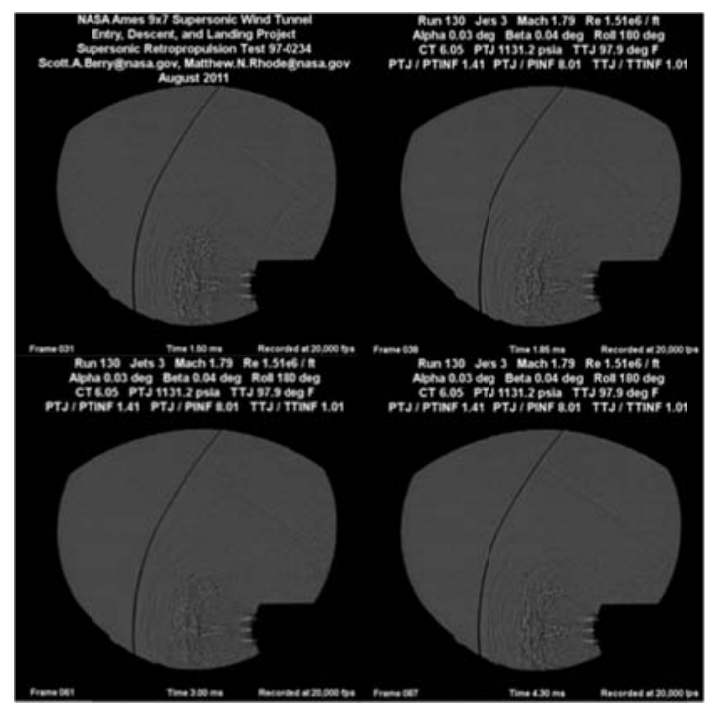

(a) Instantaneous wind tunnel shadowgraphs.
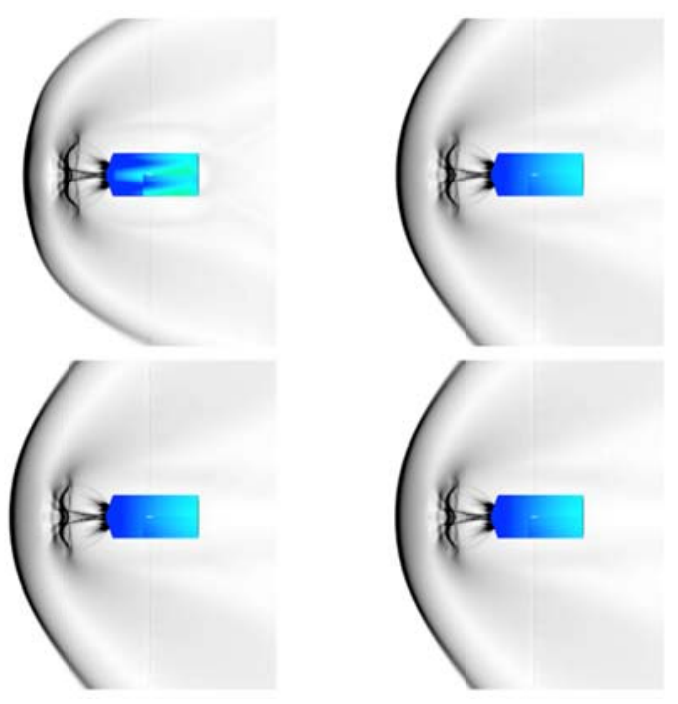

(b) Simulated shadowgraphs from DPLR flowfield solutions at four time instances. 


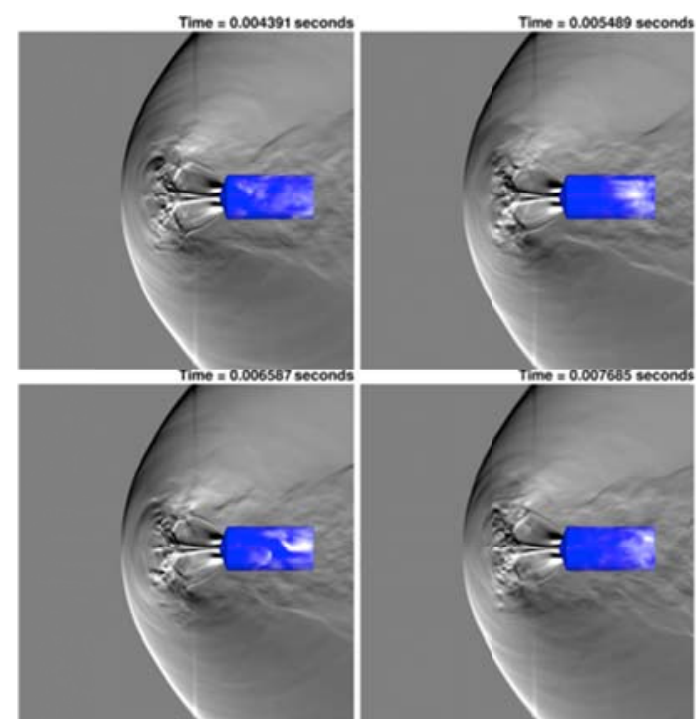

(c) Simulated schlieren from OVERFLOW flowfield solutions at four time instances.
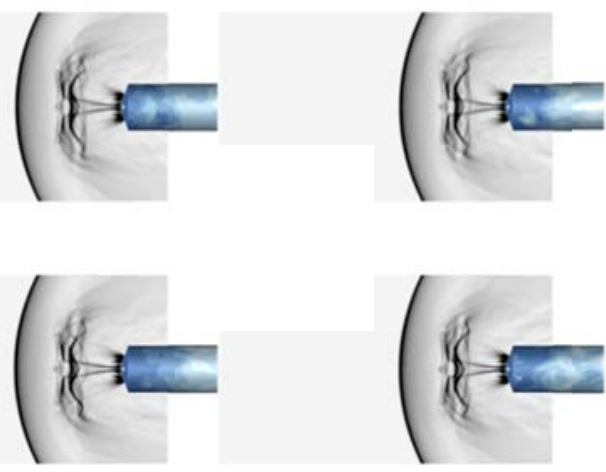

(d) Simulated shadowgraphs from FUN3D flowfield solutions at four time instances.

Figure 18: Flowfield images from wind tunnel shadowgraph and simulated shadowgraph/schlieren from CFD for Run 130. Instantaneous surface pressure coefficients are shown to qualitatively illustrate variations in time.

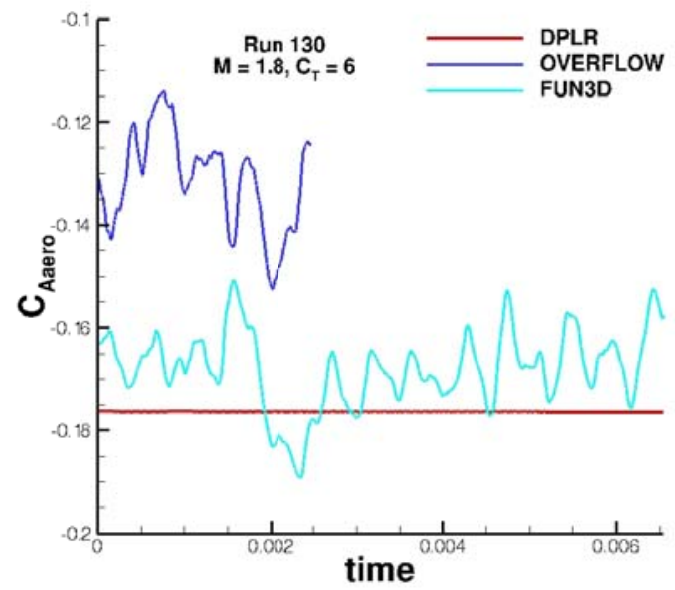

Figure 19: Aero component of transient axial force.

For Run 143, where $M=2.4$ and $C_{T}=8$, the bow shock and plume barrel shock shapes appear similar to Run 130 even though both the freestream Mach number and the thrust coefficient are stronger. The central feature between the plume barrel shocks is more pronounced for this case than in Run 130, however. Only OVERFLOW and FUN3D are displayed for this case. The CFD does a much better job for this three-nozzle case than Run 130 in predicting forebody pressure coefficients with the experiment (Figure 20 (a)). Again, both of these codes over predict the pressure coefficient at the nose, $22 \%$ by OVERFLOW and $47 \%$ by FUN3D. The pressure coefficient on the side of the model is captured by the CFD codes, varying up to $92 \%$ at the back end of the model (Figure 20 (b)). This excludes the tap at $x / L=0.65$, which appears out of family. Instantaneous shadowgraphs from the test are shown in

Figure 21 (a). Each code shows the bow shock and plume barrel shocks remain steady with variations in the free stagnation and triple point regions. Also, each code displays different characteristics for the central feature between the plume barrel shocks. The OVERFLOW schlieren images (

Figure 21 (b)) capture the smaller scales better while FUN3D shadowgraphs ( 
Figure 21 (c)) shows them more smeared out. In Figure 22 is the aero component of the axial force with computational time. From this plot the periodicity of the FUN3D solution is seen. There are many frequencies acting on this flow and it was difficult to determine the dominant frequency from the axial force for each CFD code for this case.

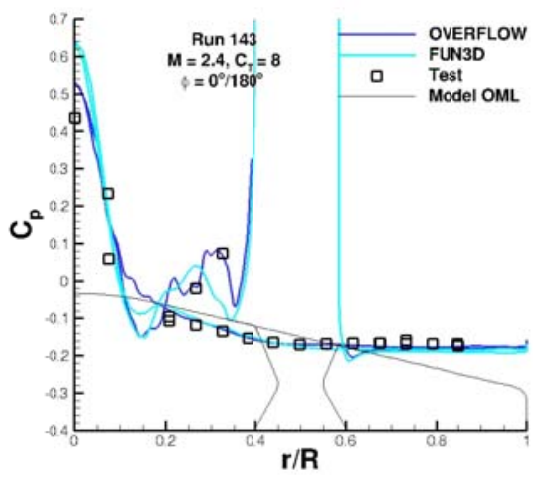

(a) Forebody

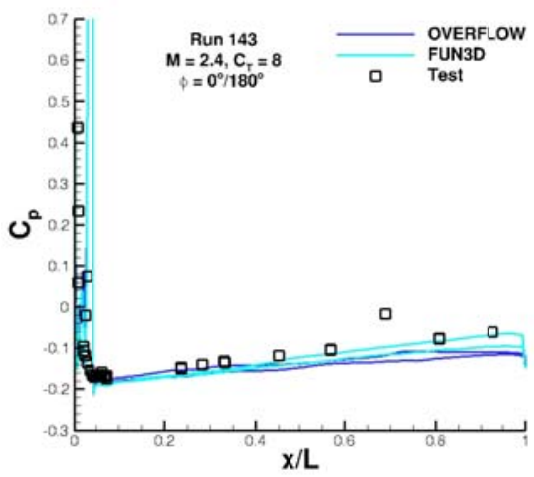

(b) Model Side

Figure 20: CFD predictions of pressure coefficients of Run 143 show excellent agreement with measurements on the forebody (a). The rise in pressure coefficient on the side (b) is captured but not the level since CFD did not model the sting.

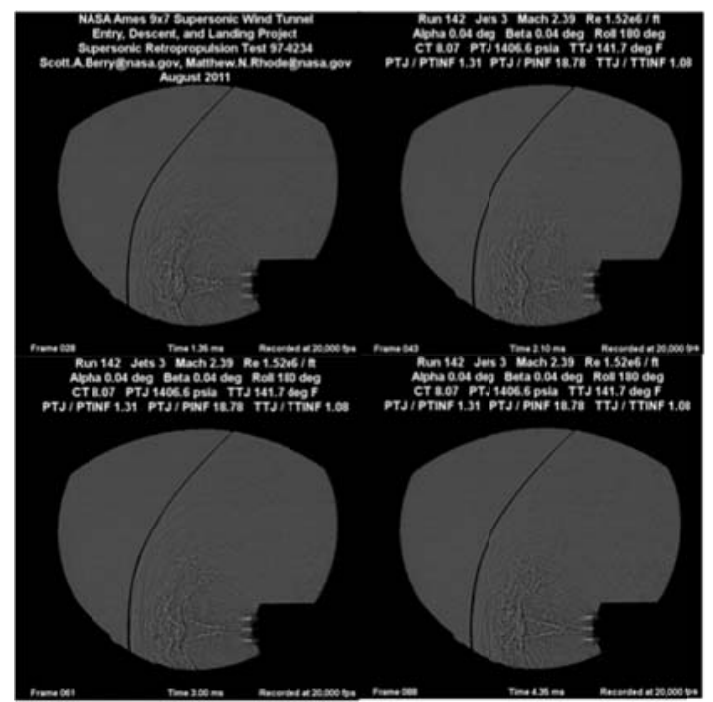

(a) Instantaneous wind tunnel shadowgraphs. 


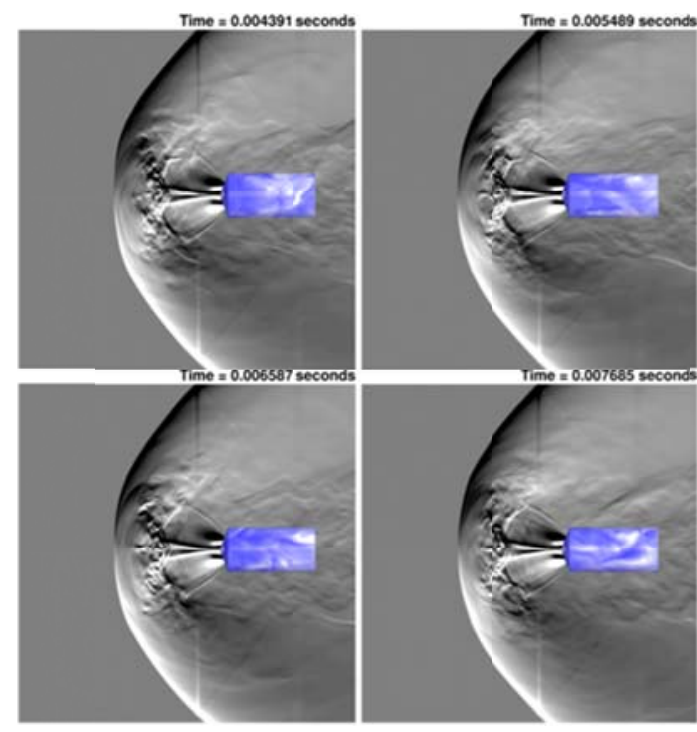

(b) Simulated schlieren from OVERFLOW flowfield solutions at four time instances.
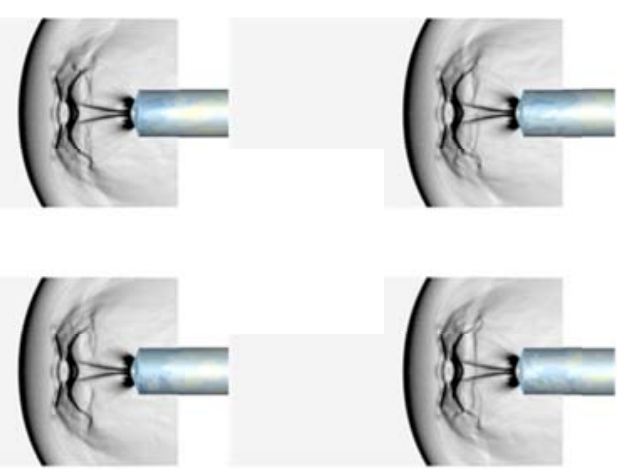

(c) Simulated shadowgraphs from FUN3D flowfield solutions at four time instances.

Figure 21: Flowfield images from wind tunnel shadowgraph and simulated shadowgraph/schlieren from CFD for Run 143. Instantaneous surface pressure coefficients are shown to qualitatively illustrate variations in time.

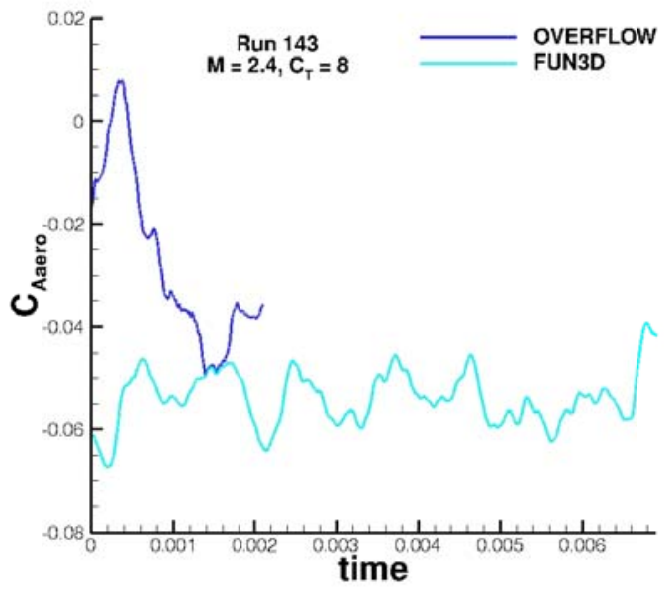

Figure 22: Aero component of transient axial force.

\section{E. Four-Nozzle Configuration}

Comparable four-nozzle runs from the LaRC UPWT test are Run 307 and 311, where $M=4.6, C_{T}=2$ and roll angle differed by $180^{\circ}$. From these runs a steadier behavior was observed than the three-nozzle cases from the same test. A periodic oscillation at the triple points was seen. The bow shock was steady and relatively close to the model. The three CFD codes had differing amounts of variation between these two runs. In the instances where the bow shock standoff distance was predicted to be much larger than observed, the pressure coefficient prediction did not suffer. This could be attributed to the plume structure shielding the model from the freestream flow. The pressure coefficient predicted by the CFD codes fell within the experimental uncertainty bars of the LaRC UPWT. ${ }^{8}$

The four-nozzle configuration results from the Ames Unitary test led to different observations than previously seen in the LaRC UPWT test. The instantaneous images for these high thrust coefficient runs show the flow to be highly chaotic. The trend of large scale flow unsteadiness at higher thrusts was not 
seen in the LaRC UPWT test due to the thrust coefficient being limited to values no higher than three due to tunnel wall interference and blockage. The Ames Unitary test was able to reach up to $C_{T}=10$.

Run 172, where $M=1.8$ and $C_{T}=8$, is unlike the previous four-nozzle cases and other cases examined in the paper. This low Mach number, high thrust coefficient case is highly unsteady and chaotic. The bow shock is unsteady and the standoff distance has large variation. The plume barrel termination shock is barely recognizable and has large deviation. Again, the only CFD codes displayed here are OVERFLOW and FUN3D. The pressure coefficient on the forebody (Figure 23 (a)) from the test is nearly constant. The two codes bound $C_{p}$ on the model side (Figure 23 (b)). As seen in

Figure 24 (a) the bow shock and plume termination shocks experience a lot of variation. Due to the limitations of the shadowgraph window viewing area the full extent of the flow field interaction is difficult to characterize. The OVERFLOW (

Figure 24 (b)) and FUN3D (

Figure 24 (c)) do experience similar variations in bow and plume termination shocks. In Figure 25 is the aero component of the axial force with computational time. From this plot the periodicity of the FUN3D solution is seen. OVERFLOW appears to indicate the same scale of unsteadiness as FUN3D. The averaging windows between the CFD codes and the test greatly differ and with such a chaotic flow, neither are probably "converged" averages. There are many frequencies acting on this flow and it was difficult to determine the dominant frequency from the axial force for each CFD code for this case.

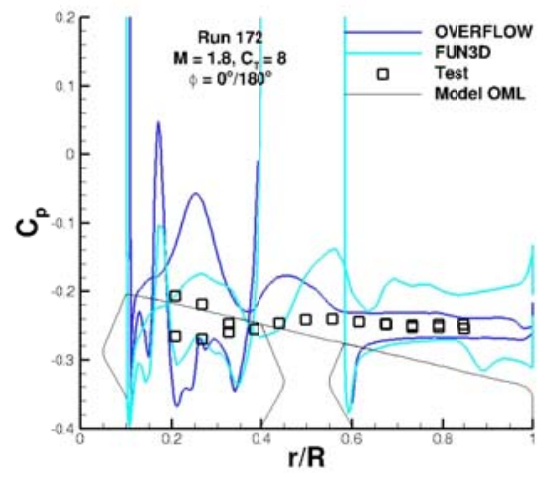

(a) Forebody

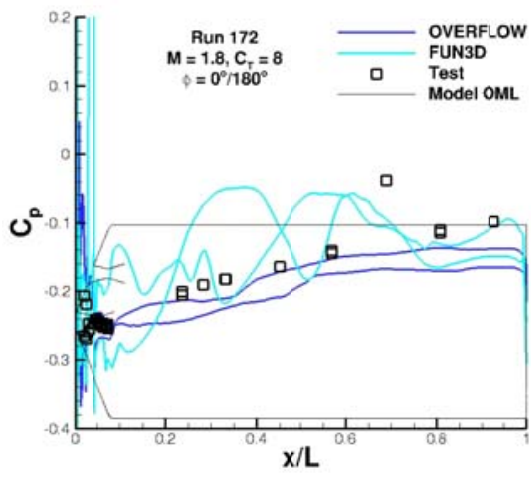

(b) Model Side

Figure 23: CFD simulations of Run 172 show excellent agreement on the forebody (a). The rise in pressure coefficient on the side (b) is captured but not the level since CFD did not model the sting.

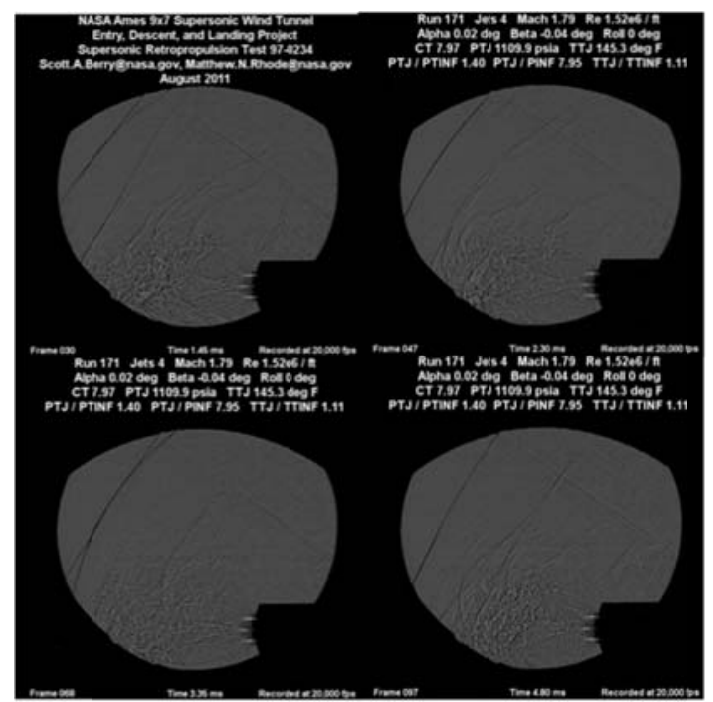

(a) Instantaneous wind tunnel shadowgraphs. 

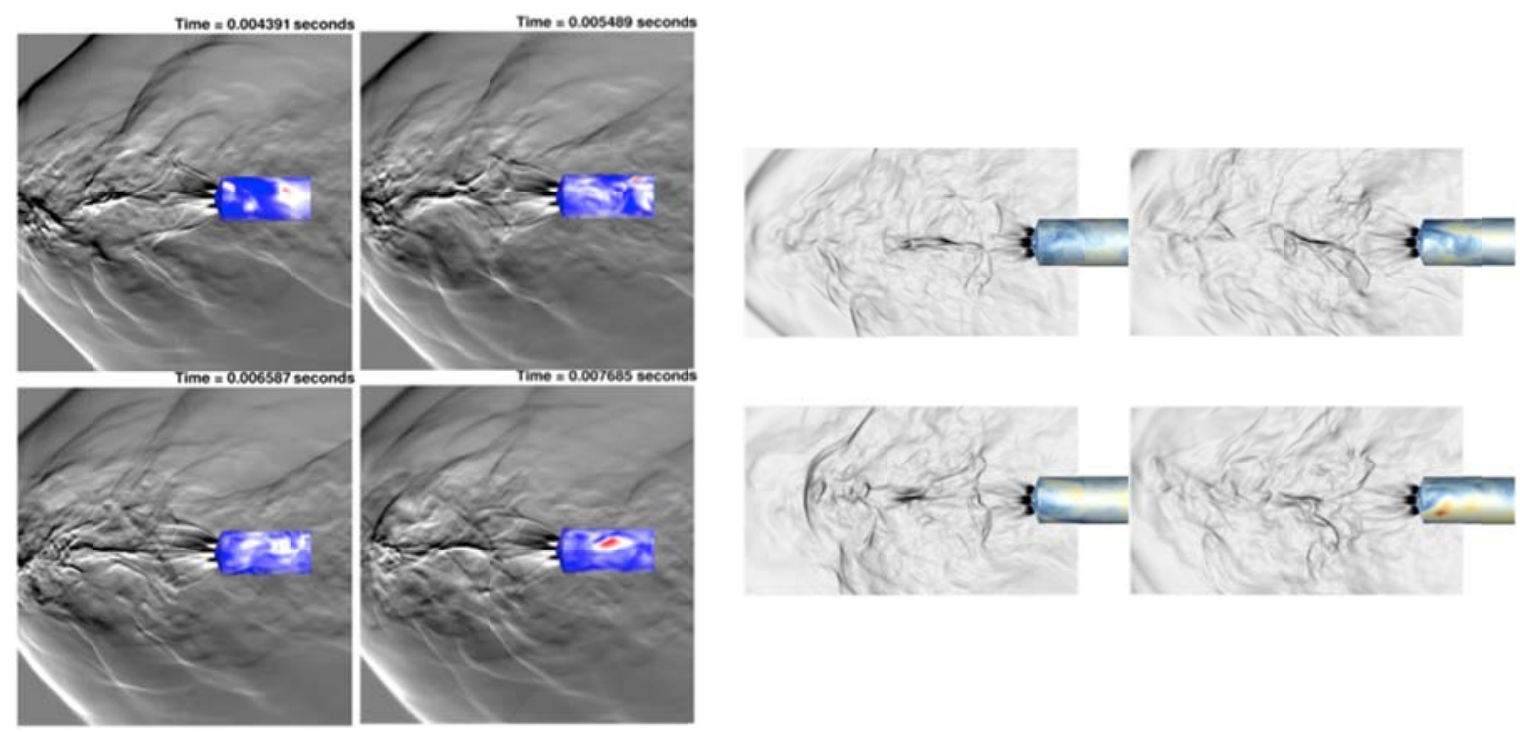

(b) Simulated schlieren from OVERFLOW

flowfield solutions at four time instances.

(c) Simulated shadowgraphs from FUN3D flowfield solutions at four time instances.

Figure 24: Flowfield images from wind tunnel shadowgraph and simulated shadowgraph/schlieren from CFD for Run 172. Instantaneous surface pressure coefficients are shown to qualitatively illustrate variations in time.

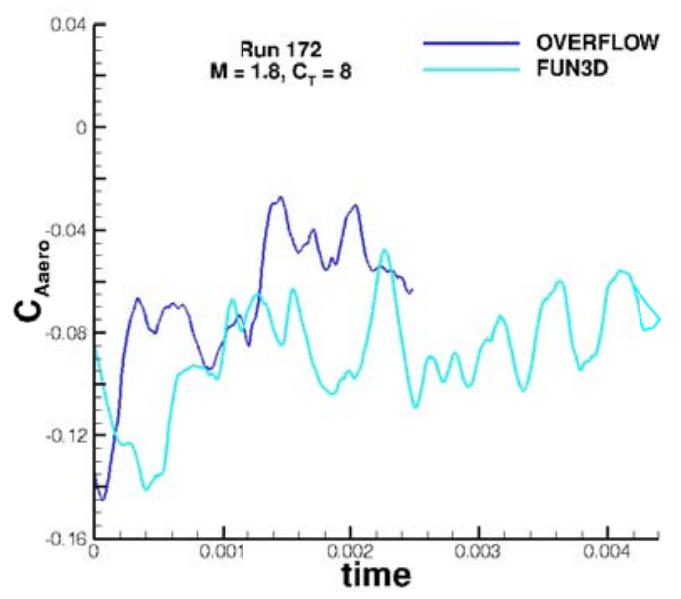

Figure 25: Aero component of transient axial force.

The Run 179, where $M=2.4$ and $C_{T}=4$, the steady nature of the lower thrust coefficient from the LaRC UPWT runs is again not present. The bow shock and plume barrel shocks are unsteady and see significant movement. The pressure coefficient on the forebody (Figure 26 (a)), however, remains fairly constant along each $\Pi$ slice, not unlike the LaRC UPWT test. The FUN3D solution appears to have insufficient averaging for pressure coefficient values. The agreement between DPLR and OVERFLOW appears to be quite good with the experiment, lying on experimental data for some locations and varying as much as $\mathbf{2 1 \%}$ for other locations. The pressure coefficient on the side of the model is also in good agreement between the DPLR and OVERFLOW and the experimental data, with up to 22\% difference (Figure 26 (b)). Again, the spikes in the DPLR line plot near $x / L=0.45$ and 0.5 are an artifact of blanking from the overset regions and are not physical. Shadowgraphs from the experiment (

Figure 27 (a)) show a lot of variation in all characteristic features including the bow shock, plume barrel shock and free stagnation regions. The simulated shadowgraphs of the DPLR flow field solutions show steadiness in the bow shock and plume barrel shocks with time ( 
Figure 27 (b)). The level of unsteadiness seen in the OVERFLOW (

Figure 27 (c)) and FUN3D (

Figure 27 (d)) simulated shadowgraphs/schlieren images is greater, where the bow shock is folding in on itself and bulging in regions not adjacent to each other. This level of unsteadiness is also seen in the test. In Figure 28 is the aero component of the axial force with computational time. From this plot the differences in the level of periodicity between the DPLR solution with the OVERFLOW and FUN3D solutions is seen. There are many frequencies acting on this flow and it was difficult to determine the dominant frequency from the axial force for each CFD code for this case.

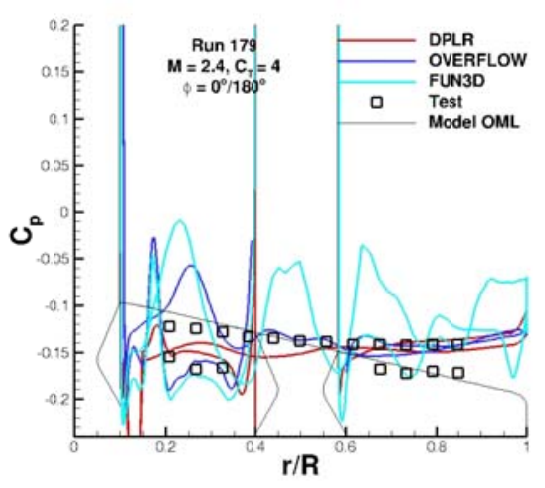

(a) Forebody

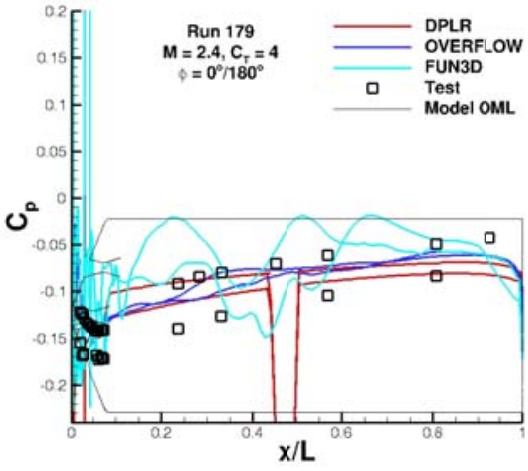

(b) Model Side

Figure 26: CFD predictions of pressure coefficients of Run 179 show excellent agreement with measurements on the forebody (a). The rise in pressure coefficient on the side (b) is captured but not the level since CFD did not model the sting.

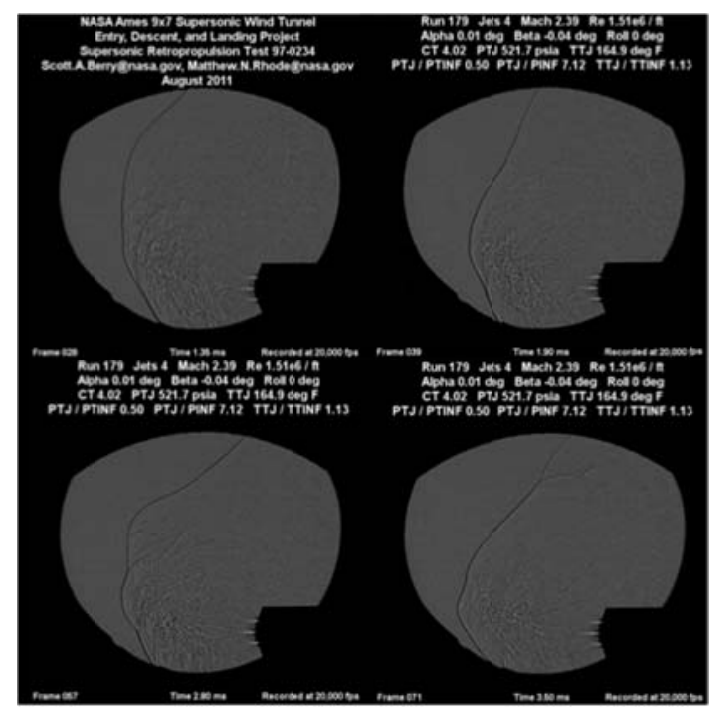

(a) Instantaneous wind tunnel shadowgraphs.
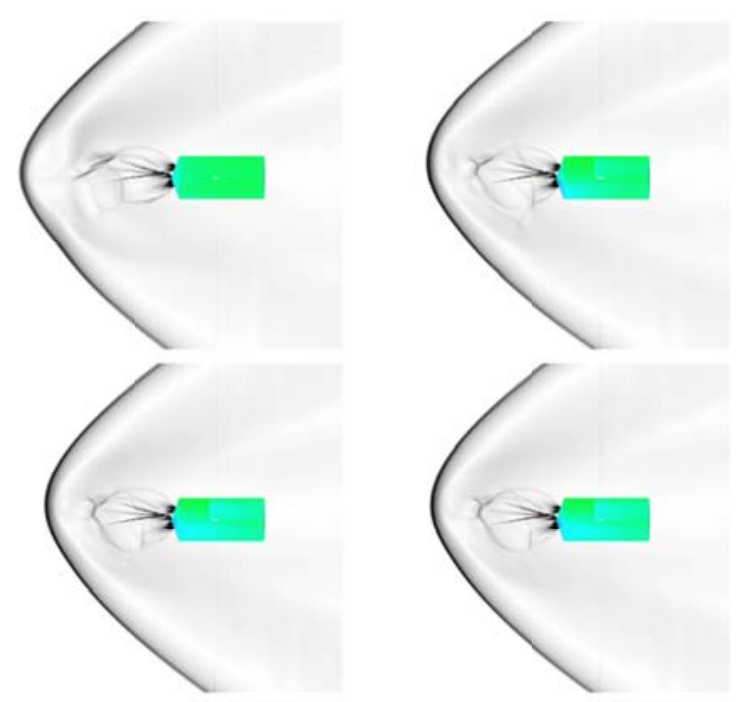

(b) Simulated shadowgraphs from DPLR flowfield solutions at four time instances. 


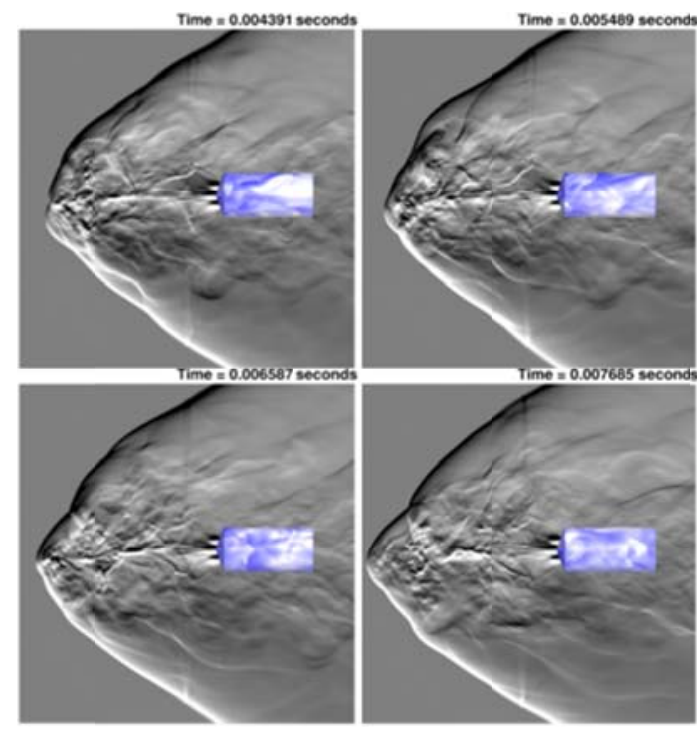

(c) Simulated schlieren from OVERFLOW flowfield solutions at four time instances.
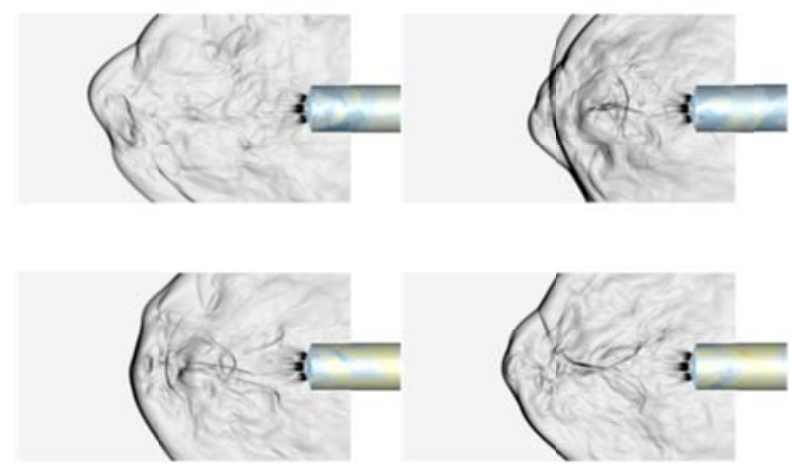

(d) Simulated shadowgraphs from FUN3D flowfield solutions at four time instances.

Figure 27: Flowfield images from wind tunnel shadowgraph and simulated shadowgraph/schlieren from CFD for Run 179. Instantaneous surface pressure coefficients are shown to qualitatively illustrate variations in time.

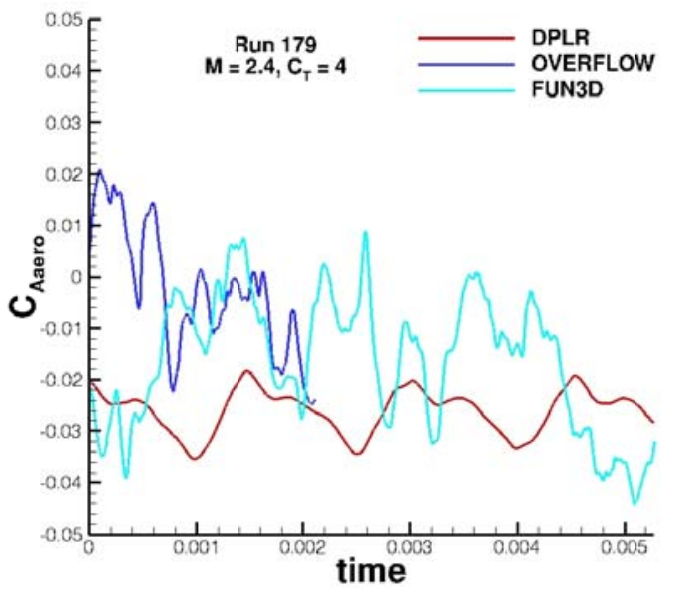

Figure 28: Aero component of transient axial force in DPLR and FUN3D.

\section{Summary}

The present paper was focused on the prediction of supersonic retropropulsion flow fields for high thrust coefficients $\left(C_{T}>3\right)$ of single- and multi-nozzle configurations at two freestream Mach numbers $(1.8$ and 2.4) - conditions tested in the second of two tests in the Ames Unitary wind tunnel in August 2011. The Navier-Stokes codes DPLR, OVERFLOW, and FUN3D, with best practices established during simulations of the previous SRP wind tunnel test in the LaRC UPWT, were exercised in the present work.

Surface pressure measurements and shadowgraphs from the test have been used to evaluate the flow prediction tools. All codes predict the baseline smooth (no-nozzle) configuration pressure measurements to within $\pm 1.8 \%$. An analysis of incorporating the sting into the simulations to determine its influence on predicted flow fields was performed. The sting can be said to have some influence on the surface pressure at the aft end of the model. The single-nozzle configuration shows a periodic oscillation in the triple point region yet little variation in the bow shock and plume termination shock features. The CFD codes predicted 
different frequencies of the system, but all codes predicted the pressure measurements to within $24 \%$. The three-nozzle configuration also has a periodic oscillation in the triple point region and little variation in the bow shock and plume termination shock features. This is counter to what was seen in the previous LaRC UPWT test, where at low thrust coefficients $\left(C_{T} \leq 3\right)$ the flow field was chaotic and saw a lot of movement in the shock locations at different instances in time. Meanwhile, the four-nozzle configuration at higher thrust coefficients $\left(C_{T}>3\right)$ was highly unsteady and chaotic. All features, including the bow shock, plume barrel shocks, free stagnation and triple point regions had large variations in time. The CFD codes were able to capture the large variations in all the flow features with computational time. The prediction of surface pressure coefficient for the four-nozzle cases did not agree as well with test data as with the steadier cases.

Between the codes, the largest differences were in the frequencies and unsteadiness predicted. The turbulence models used by each code were different and could attribute to these differences. DPLR showed the lowest level of unsteadiness and used the RANS SST turbulence model with vorticity-based production term. The OVERFLOW code used DES with SST submodel, while FUN3D used DES with SA submodel. Each of these turbulence models generates varying levels of eddy viscosity, which adds dissipation to the solutions making them steadier. ${ }^{9}$ In general, the RANS models were steadier than the DES. The ability to properly capture the unsteady effects appeared to have little effect on comparing with the time-averaged surface pressures.

Future study in SRP flows would involve further examining the Ames Unitary test data across a larger range of thrust coefficients and the effect of non-zero angle of attack. Also, further examination of unsteadiness would be warranted as well. Some of the unsteadiness is driven by differences in numerical dissipation employed by the various schemes implemented in the three codes. One way to get an understanding of this would be to refine the grids to see if that would increase the level of unsteadiness. Yet another way would be to apply a "low dissipation" scheme. Obtaining a laminar solution from each CFD code would inform the effect of turbulence modeling on the steadiness obtained. It would be important to assess how the unsteadiness could affect the vehicle stability as well.

\section{References}

${ }^{1}$ Braun, R. D. and Manning, R. M., "Mars Exploration Entry, Descent, and Landing Challenges," Journal of Spacecraft and Rockets, Vol. 44, No. 2, Mar-Apr 2007.

${ }^{2}$ Steinfeldt, B. A., Theisinger, J. E., Korzun, A. M., Clark, I. G., Grant, M. J., and Braun, R. T., "High Mass Mars Entry, Descent, and Landing Architecture Assessment," AIAA Paper 2009-6684, Sep 2009.

${ }^{3}$ Zang, T. A., Dwyer-Dianciolo, A. M., Kinney, D. J., Howard, A. R., Chen, G. T., Ivanov, M. C., Sostaric, R. R., and Westhelle, C. H., "Overview of the NASA Entry, Descent and Landing Systems Analysis Study," AIAA Paper 2010-8649, Aug 2010.

${ }^{4}$ Edquist, K. T., Dyakonov, A. A., Korzun, A. M., Shidner, J. D., Studak, J. W., Tigges, M. A., Kipp, D. M., Prakash, R., Trumble, K. A., and Dupzyk, I. C., "Development of Supersonic Retro-Propulsion for Future Mars Entry, Descent, and Landing Systems," AIAA Paper 2010-5046, Jun 2010.

5 Korzun, A. M. and Braun, R. D., "Performance Characterization of Supersonic Retropropulsion Technology for High-Mass Mars Entry Systems," Journal of Spacecraft and Rockets, Vol. 47, No. 5, SepOct 2010.

${ }^{6}$ Korzun, A. M., Braun, R. D., and Cruz, J. R., "Survey of Supersonic Retropropulsion Technology for Mars Entry, Descent, and Landing," Journal of Spacecraft and Rockets, Vol. 46, No. 5, Sep-Oct 2009.

${ }^{7}$ Trumble, K. A., Schauerhamer, D. G., Kleb, W. L., Carlson, JR., Buning, P. G., Edquist, K. T., and Barnhardt, M. D., "An Initial Assessment of Navier-Stokes Codes Applied to Supersonic RetroPropulsion," AIAA Paper 2010-5047, June 2010.

${ }^{8}$ Schauerhamer, D. G., Trumble, K. A., Kleb, W. L., Carlson, JR., Edquist, K. T., "Continuing Validation of Computational Fluid Dynamics For Supersonic Retropropulsion," AIAA 2012-864, January 2012.

${ }^{9}$ Kleb, W. L., Carlson, JR., Buning, P. G., Berry, S. A., Rhode, M. N., Edquist, K. T., Schauerhamer, D. G., Trumble, K. A., Sozer, E., "Toward Supersonic Retropropulsion CFD Validation," AIAA 2011-3490, June 2011.

${ }^{10}$ Berry, S. A., Rhode, M. N., Equist, K. T., Player, C., "Supersonic Retropropulsion Experimental Results from the NASA Langley Unitary Plan Wind Tunnel," AIAA 2011-3489, June 2011.

${ }^{11}$ Trumble, K. A., Schauerhamer, D. G., Kleb, W., Carlson, J-R, Edquist, K., "Analysis of Navier-Stokes Codes Applied to Supersonic Retro-Propulsion Wind Tunnel Test," IEEE, paper \#1471, March 2011. 


\footnotetext{
${ }^{12}$ Wright, M.W., White, T., and Mangini, N., "Data Parallel Line Relaxation (DPLR) Code User Manual Acadia - Version 4.01.1,” NASA/TM-2009-215388, October 2009.

${ }^{13}$ Anderson, W.K. and Bonhaus, D.L., "An Implicit Upwind Algorithm for Computing Turbulent Flows on Unstructured Grids," Journal of Computational Physics, Vol. 128, No. 2, 1996, pp. 391-408.

${ }^{14}$ Anderson, W. K., Rausch, R. D., and Bonhaus, D. L., "Implicit/Multigrid Algorithm for Incompressible Turbulent Flows on Unstructured Grids," Journal of Computational Physics, Vol. 128, No. 2, 1996, pp. 391-408.

${ }^{15}$ Buning, P. G., Jespersen, D. C., Pulliam, T. H., Klopfer, G. H., Chan, W. M., Slotnick, J. P., Krist, S. E., and Renze, K. J., "Overflow User's Manual," NASA Langley Research Center, Hampton, VA, 2002.

${ }^{16}$ MacCormack, R.W. and Candler, G.V., "The Solution of the Navier-Stokes Equations Using GaussSeidel Line Relaxation," Computers and Fluids, Vol. 17, No. 1, 1989, pp. 135-150.

${ }^{17}$ Yee, H.C., "A Class of High-Resolution Explicit and Implicit Shock Capturing Methods," NASA TM 101088, Feb. 1989.

${ }^{18}$ Menter, F.R., "Improved Two-Equation $\kappa-\omega$ Turbulence Models for Aerodynamic Flows," NASA TM 103975, October 1992.

${ }^{19}$ Edwards, J. R., "A Low-Diffusion Flux-Splitting Scheme for Navier-Stokes Calculations," Computers \& Fluids, Vol. 26, 1997, pp. 653-659.

${ }^{20}$ Van Albada, G. D., B. Van Leer, B., and Roberts, W. W., (1982), "A Comparative Study of Computational Methods in Cosmic Gas Dynamics," Astron. Astrophysics, Vol. 108, 1982, p. 76-84.

${ }^{21}$ Lynch, C.E. and Smith, M.J., "Hybrid RANS-LES Turbulence Models on Unstructured Grids," AIAA Paper 2008-3854, June 2008.

22 Tramel, R., Nichols, R., and Buning, P.G., "Addition of Improved Shock-Capturing Schemes to OVERFLOW 2.1," AIAA Paper 2009-3988, June 2009.

23 Nichols, R.H., Tramel, R.W., and Buning, P.G., "Solver and Turbulence Model Upgrades to OVERFLOW 2 for Unsteady and High-Speed Applications," AIAA Paper 2006-2824, June 2006.

${ }^{24}$ Rhode, M. N., and Oberkampf, W. L., "Estimation of Uncertainties for a Supersonic Retro-Propulsion Model Validation Experiment in a Wind Tunnel," Accepted for Publication to 42nd AIAA Fluid Dynamics Conference, New Orleans, LA, June 2012.

${ }^{25}$ Berry, S. A., Rhode, M. N., Edquist, K. T., "Supersonic Retropropulsion Experimental Results from the NASA Ames 9- x 7-Foot Supersonic Wind Tunnel," Accepted for Publication to 42nd AIAA Fluid Dynamics Conference, New Orleans, LA, June 2012.
} 